\title{
Social Agents to Enable Pervasive Social Networking Services
}

\section{Iosif Alvertis', Evmorfia Biliri², Fenareti Lampathaki ${ }^{3}$, Dimitris Askounis ${ }^{4}$}

${ }^{1}$ National Technical University of Athens, School of Electrical \& Computer Engineering, Athens, Greece, alvertisjo@epu.ntua.gr

${ }^{2}$ National Technical University of Athens, School of Electrical \& Computer Engineering, Athens, Greece, ebiliri@epu.ntua.gr

${ }^{3}$ Suite5 Data Intelligence Solutions Limited, Limassol, Cyprus, fenareti@suite5.eu

${ }^{4}$ National Technical University of Athens, School of Electrical \& Computer Engineering, Athens, Greece, askous@epu.ntua.gr

Received 22 November 2016; received in revised form 11 October 2017; accepted 27 December 2017

\section{Abstract}

Over the past decade, the way people communicate, share content and eventually conduct business has been disrupted by social networking platforms. By bringing together pervasive social computing with social networking under a common modelling methodology and common social rules based on human activities in accordance with the Activity Theory, our work aims at contributing to the standardisation of the Social Web in a way that is independent from proprietary solutions, interoperable and semantically rich for applications beyond social networking. The concept of a Social Agent is introduced as a set of micro-services to handle digital traces of human activities for further analysis, respecting both users' privacy and the business models of companies that build personalised services or applications in the web. In this paper, the landscape of social networking, social pervasive computing and social agency is presented in detail, the research questions driving our work are explained and the fundamentals of a Social Agent along with the pervasive social network it may build are set. A quantitative evaluation based on the Technology Acceptance Model methodology is implemented on a sample of 200 interviewees, evaluating the concepts of the Social Agent, while its technical feasibility is proven and presented through a prototype.

Keywords: Social agent, Pervasive social networking, Social web, Close-distance communication, Human activities, Digital traces, Social agent, Activity theory, Interoperability, Privacy 


\section{Introduction}

Nowadays, people spend a great amount of time in social media [72], where generated data and metadata reveal their consuming behaviour and show preference over certain products and services. Those users' digital traces and personal data spur tremendous opportunities for new personalised services and applications, evolving into assets for e-businesses that leverage them to enhance users' experience [72]. Such an influx of personal data from social networking services [76], combined with sensor-based data coming from mobile devices, have enabled a progressive transition from social networking to pervasive social computing [50]. However, digital social networking platforms have evolved into fragmented centralised solutions [7] with strong contextual characteristics [31], without sharing the generated knowledge from users' data with other stakeholders, not even with the owners of the data [7].

The multi-sided business models of prevailing social media platforms have the downside that the emerging data economy [9] allows organizations to build their business on selling or reselling users' personal data [19]. Such privacy issues have emerged both at business [15] and governmental level (Site 9), while people have not perceived the value of their data or the severity of such privacy violation incidents [57]. In parallel, even when companies have officially integrated social media management in their strategy [48], they have limited access to that customer knowledge which is restricted to the business services provided by the social media platforms to them. Thus, the dependence of the Social Web on centralised solutions and its inextensibility in areas outside social networking, due to lack of interoperability and standardisation, are the main problems to address in our research.

The business and technical obstacles towards an interoperable, open social web may be summarised in five main points [23], [54]: (a) API policies and limitations regarding collecting, storing and processing data from social media platforms [2]; (b) development and hosting costs for decentralised social media services that the majority of users are not willing to pay, due to modern ad-based business models they are accustomed to; (c) time and effort required from users to move to new social networking solutions; (d) lack of users' trust towards third party developers to set up alternative solutions or meta-platforms [30], without any clear additional value for users apart from increased data privacy and ownership; and (e) lack of standards to integrate social web [2]. The discussion of leaving existing social media platforms merely due to privacy concerns has become irrelevant lately [69]; users will move to another solution - or will use an additional one - only if they consider it as a new offering, or if their social connections move to another platform first [6].

Our work introduces Activity Theory as an umbrella theory to unify pervasive social computing with social networking under a common modelling methodology and common social rules. The goal of our research is to standardise Social Web in a way independent from proprietary solutions, interoperable and semantically rich for applications outside social networking, by designing, evaluating and prototyping a novel approach based on human activities in the area of pervasive social networking. The proposed solution is built on the concept of a Social Agent and it is a user-centric solution based on reusing digital traces to create social connections ubiquitously, without violating users' privacy or destroying economic value for companies that build personalised services or applications. Briefly, a Social Agent is capable of integrating data, digital traces and meta-data from various resources under Activity Theory, analysing them under the principles of hierarchy and internalisation as described in the relative theory, and then setting up pervasive social and privacy rules in order to enable agent-driven social interactions with other Social Agents or pervasive services in proximity, by exchanging semantically rich and interlinked messages formatted again under Activity Theory.

The three main research questions that drive current work, as explained in the analysis of section 2.4 , are the following (see Table 1): (a) How digital traces coming from different services and devices may be modelled in order to be combined with content from social media, in an interoperable layer of personal data, to enable the exchange of social networking messages across decentralised services and devices, without losing the semantic power of that information. (b) How a Social Agent may be able to represent its owner under a strong privacy framework, without violating users' privacy, in order to allow the exchange of the aforementioned messages and establish meaningful social connections pervasively. (c) Finally, as long as various research work and industrial solutions have failed to get the traction needed, the question is what methodology may be developed to measure users' acceptance over pervasive social networking, without having an initial community, in order to evaluate new concepts and features of pervasive social networking that are not clear to the users beforehand.

Table 1: Research questions on pervasive social networking

\begin{tabular}{|l|l|l|}
\hline $\begin{array}{l}\text { Identified Categories of } \\
\text { Gaps }\end{array}$ & ID & Research Question \\
\hline Social Data Modelling & RQ1 & $\begin{array}{l}\text { How should digital traces be modelled in interoperable, semantically-rich social } \\
\text { messages that will enable pervasive social networking? }\end{array}$ \\
\hline Pervasive social & RQ2 & $\begin{array}{l}\text { How should pervasive social networking implement social agency, without } \\
\text { violating users' privacy? }\end{array}$ \\
\hline Social acceptance & RQ3 & $\begin{array}{l}\text { How may we measure users' acceptance in pervasive social networking } \\
\text { without having a community of users? }\end{array}$ \\
\hline
\end{tabular}


The methodological steps to build and evaluate a Social Agent were the following (see figure 1): In phase one, there was an in-depth analysis of the landscape of Social Networking, Pervasive Computing and Social Agency (documented in Chapter 2), followed by the definition of the research questions and the introduction of the concept and the fundamentals of a Social Agent (as described in Chapter 3 in its final version). As a last step in that phase, a quantitative evaluation based on the TAM methodology was implemented on a sample of 200 interviewees, evaluating both the generic concept and the specific functionalities of a Social Agent (see section 4.1). In phase two, based on the outcomes of the quantitative analysis, the concept of the Social Agent was refined and then it was modelled under a relative methodology (available in Chapter 3 ). Then its technical feasibility was tested with the development of an initial prototype and it was tested qualitatively against 20 users who synced their data and used the Social Agent (see section 4.2). In the final phase, the outcomes of our research and its contribution in the domain of pervasive social networking were generated (Chapter 5), while the future steps in the research roadmap of a Social Agent were developed to put the concept towards improved usage acceptance (Chapter 6).

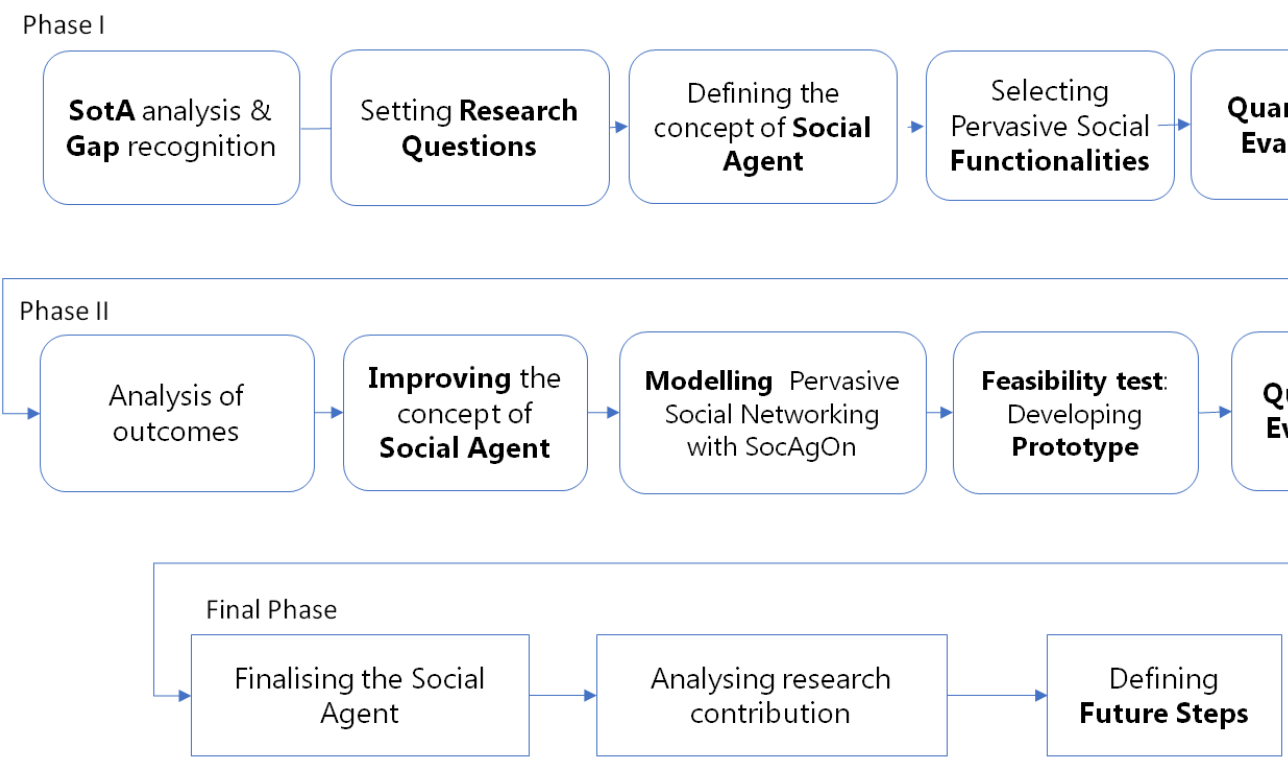

Figure 1: The methodology for developing a pervasive social agent

\section{Related Work}

Online Social Network Services (oSNS) are "web-based services that allow individuals to (1) construct a public or semi-public profile within a bounded system, (2) articulate a list of other users with whom they share a connection, and (3) view and traverse their list of connections and those made by others within the system", [10] to enable "communication of participants through message exchange"' [21]. However, since 2008 people have become hyperconnected over mobile devices and faster mobile networks, thus there is the need to unbind the definition of SNS from the Web as the main medium of socialising, and focus on the activities of the users that create social connections among people in the physical and digital environment; thus, in a more general definition, Social Networks are "new applications and services that facilitate collective action and social interaction online with rich exchange of multimedia information and evolution of aggregated knowledge" [51].

From an evolutionary perspective, digital social networking is now going through its fourth phase (see figure 2): from content-based applications to media-based platforms, then to location aware services, and lately to contextually aware social networking services [31]. This latest phase of social networking puts the exchange of a social object among users in the centre of social networking (Site 1) to establish permanent or temporal ties among people and form social networks even based on contextual information. To this direction, the widespread adoption of handheld devices, wearables and users' intrinsic social nature have been combined with context-aware interactions, known as Pervasive Social Networking. Pervasive Social Networks (PSN) act on top of or in parallel with existing Social Networking Platforms and complement virtual interactions with physical ones, by connecting users in social and physical proximity [50], inside or outside their current social relations. 


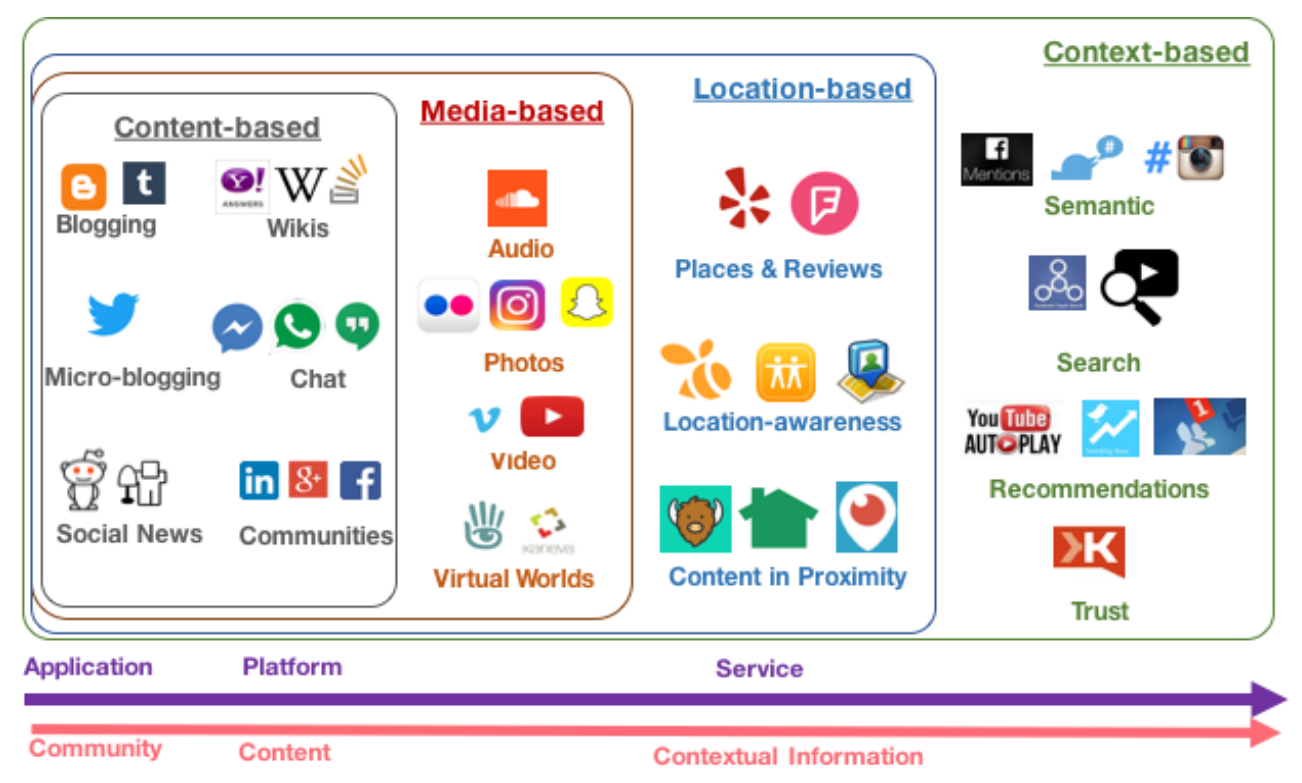

Figure 2: The transition of social networking services towards context, based on Irfan et al. [31]

Pervasive Social Networking combines characteristics from Pervasive Computing with the goals of Digital Social Networking. Such PSN services should handle pervasive messages to establish social connections based on the social goals and motives of the users, a behaviour that implies the presence of agency. In that direction, this chapter analyses three main areas: (a) the current landscape of social networking, with alternative architectures and models, to realise how current designs have hindered interoperability in the Social Web (i.e. section 2.1), (b) the domain of Pervasive Social Computing and why it fails to perceive Pervasive Social Networking as an independent solution of social networking (i.e. section 2.2), and (c) the area of Social Agency that models and implements social rules and motivations (i.e. section 2.3) as an enabler for new forms of social networking interactions. Finally, section 2.4 summarises the value chain of pervasive social networking and identifies the gaps where our research focuses on.

\subsection{Social Web}

The fragmentation of the Social Web in centralised silos of users' data, driven by social media platforms, is as Tim Berners-Lee states [49] a social problem rather than a technological one, as people choose to use mainly the dominant solutions even if the Web is already decentralised technologically. To address the underlying limitations, research was focused on two main tracks (Figure 3): (a) provide alternative architectures for social networking platforms, to allow users to connect using a single digital identity (i.e. left side of figure), and (b) agree on a common modelling for the Social Web, towards standardisation and interoperability (i.e. right side of figure).

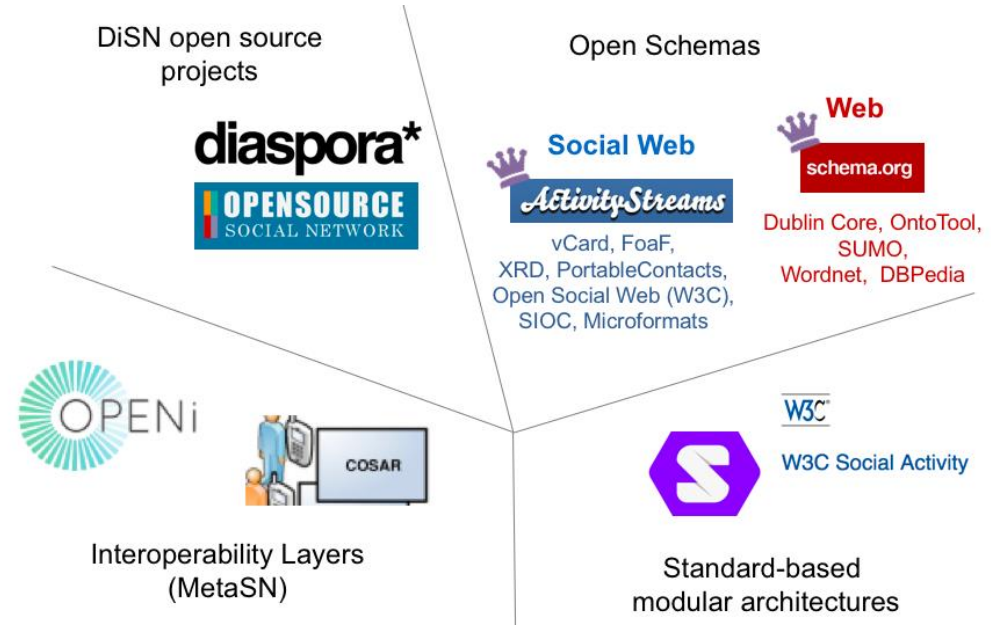

Figure 3: The different areas of research for the Social Web, under modelling and architectural standardisation

Architecture-wise, relative work [29] has identified three types of Distributed Social Networks (DSN) as alternatives to existing centralised solutions accordingly to their degree of decentralization: (i) distributed if the clients use different protocol, (ii) federated if they use the same protocol and (iii) meta-social networks if they operate in parallel to existing 
social networks. However, the main criteria for classifying the alternative architectures of a Social Network Platform should be what is the trusted point where users may get access to their social data and access their social network, in the following way (see Figure 4): (a) Centralised Social Networks (CeSN) are based on a unique point of access to the network (Figure a), and it is the architecture that social networking platforms typically follow at the moment. (b) Distributed Social Networks (DisSN) allow clients to store and access their data through different platforms, while there is communication among these central points (Figure 2b); e.g. a user should be able login to Facebook and find content and connections from Twitter. (c) A Meta-Social Network (MetaSN) connects different social networks under a unified, interoperable layer (Figure 2c), as middle layer that connects different platforms. (d) Federated or Peer-to-Peer (P2P) Social Networks (P2PSN) allow users to spread their data across different clients, encrypted, without needing any central point of storage to make the network operational (Figure 2d). (e) Mesh Social Networks (MeshSN) are highly distributed networks with special routing technology to exchange messages both as receivers and routers (Figure 2e). Finally, (f) Hybrid Social Networks combine characteristics from different architectures. Overall, for architectures (a-c) it is visible that if CeSNs agreed on a common protocol and common interfaces, they would involve into DisSNs and people would access unique content across different platforms.

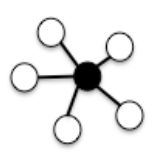

CeSN1

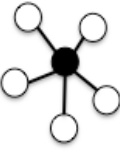

CeSN2

(a)

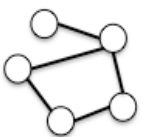

FedSN1

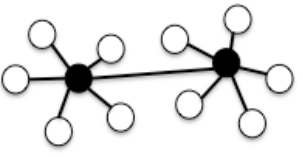

DisSN

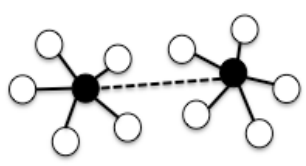

MetaSN

(c)

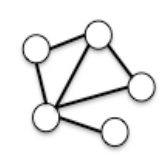

FedSN2

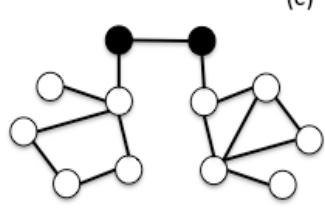

MeshSN

(d)

(e)

Figure 4: Examples of different types of social networks

Data-wise, there is no agreement yet under the relative W3C group (Site 5) on standardising Social Web, while the plethora of APIs has created a confusion of semantics in SNPs. The most important schemas in the direction of standardisation are:

1. ActivityStreams (Site 6) is a protocol describing user activities over social web services. The protocol introduces the basic syntax for a user activity, in the form of \{subject\} \{verb\} \{object\}, and may be augmented with contextual information such as the location of the activity or the mood of the user. A suggested dictionary for the verbs that have been recognised over various platforms has been developed, while its second version is under standardisation discussions by W3C. Practically, ActivityStreams is used in system notifications and is integrated in proprietary schemas (e.g. plugins of web frameworks).

2. (Site 7) it is promoted by Google as a standard to describe resources on websites to improve semantics and indexing on search results, and it has a dictionary of objects with predefined properties.

3. There are various other proposed protocols in Social Web, such as vCard (Site 14), FoaF (Site 15), XRD (Site 16), OpenSocial transformed into W3C Social Web Activity (Site 17), SIOC project (Site 18) and Microformats (Site 19), and many others to describe objects in general, like Dublin Core (Site 20), SUMO (Site 21), WordNet (Site 22), and DBPedia (Site 23).

Implementation-wise, in the direction of identifying holistic solutions towards standardising the way social networks are developed (i.e. both architecturally and data-wise), three main approaches may be found:

1. Open Implementations: Diaspora (Site 11) and Open Source Social Network (Site 12) are two well-known industrial social networking platforms that focus on distributed social architectures (DiSN) by making the source code of the platforms reusable, rather than interoperable with existing social networking platforms.

2. Interoperability layers: Progress has been made on meta-platforms (MetaSN) as hybrid approaches to integrate the Social Web with social applications [2], [11]. The platforms suggest a common schema, based on modelling standards (I.e. ActivityStreams and (site 7)), where they map data across different platforms, to form MetaSNs. 
3. Standard-based modular architectures: Solid [49] (Site 8) works on modular and extensible applications, based on W3C standards, which may be reused and interconnected in order to build social media platforms.

Overall, semantic dictionaries to describe objects even outside the Web and a behavioural framework to integrate different domains are still missing from schemas of the Social Web. For alternative architectures, the main gap is the lack of motivation from users to move to a new solution and leaving existing convenience and ease of use; users seek playfulness when interacting with their social interactions and would not move to another platform just for technical or architectural reasons [16]. In other words, in order to change the way people socialise over technology nowadays, just redesigning the technological backbone may not be enough. This is where pervasive schemas may contribute with broader concepts and pervasive social interactions that engage users' interest with new offerings and functionalities.

\subsection{Pervasive Social Networking}

Considering the unwillingness of social media platforms to collaborate towards an open Social Web and the stagnation of social networking users with existing solutions, the domain of Pervasive Social Networking is a promising research area where pervasive services may be combined with hardware and social networking services towards more interoperable social networking interfaces. Pervasive Social Networking is still social networking with strong behavioural data that can reveal the context of a human activity, the motives and the factors that affect a user's action or decision, or per Schuster D. et al. [64] pervasive social context of an individual is the set of information that arises out of direct or indirect interaction with people carrying sensor-equipped pervasive devices connected to the same social network service. Pervasive Social Networking is a specialised area of Pervasive Social Computing, working on ubiquitously establishing social connections among users based on contextual data, while Pervasive Social Computing is a broader research area focusing on the social dimensions of contextual data used in pervasive interactions. Thus, under that perspective, the modelling and the usage of contextual social data are examined in this section, progressively from pervasive social computing to pervasive social networking, to identify common points with social networking modelling and unveil any behavioural theory that research in social web has neglected.

In Pervasive Social Computing, the social context is mainly perceived as a subcategory of the broader user context, separated from the physical context, used to improve users' experience ubiquitously while users interact with computer systems. Under the Organizational Context [39], the social context has the form of personal context, defining user's preferences, profiles and interests. In the User Profile Ontology with Situation-Dependent Preferences Support (UPOS) [67], the user context has been separated from user's preferences, while in Context-Aware Social Networking Services (SNSs) [42] the user profile, habits, preferences and attitude are under a distinct category named logical contextual information. Later, research work on pervasive computing started to distinguish user's context from social context. For the ontology of Pervasive Social Computing [75], the social context is organised under behavioural awareness for social signal processing, community awareness for metadata collected from social networking platforms, and content awareness for data collected from social media. Under the Well-being Context [3], the social context is a seperate category that describes the neighbourhood and the community where the user is located. Finally, the ST(i)PI taxonomy [64] splits data that come from social networks in two categories: context on data collected directly from social networks (e.g. content) and context on individuals, groups and communities that a user belongs to or interacts with.

In Pervasive Social Networking, context is perceived as an enabler for social networking, where four main approaches have been identified (Figure 5): (a) There are contextual enhancements on existing social networking solutions, to improve social interactions and deliver improved features, with Facebook News Feed and Instagram Feed to adapt already to users' profiles and devices. (b) There are platforms acting as middleware to collect and reuse social networking information from existing platforms, to boost social interactions or improve application behaviour on third party applications, but not for social networking purposes [43]-[46]; e.g. combining social network proximity with location context to improve interactions in physical space through devices [50]. (c) Contextual information from existing social networking is used for further social networking by third party systems [3], [42], [52], [64], [75], which nevertheless are not standalone solutions as they depend on the source of that information. Finally, (d) there are dedicated solutions for pervasive social networking, partially based on existing platforms or even completely independent from them, on dedicated hardware to boost close-distance communication [26], [43], (Site 2), (Site 3); e.g. smart textiles show users' similar interests when users touch each other's T-shirt [33].

The capabilities of recognising and adapting to human behaviour are important dimensions of such pervasive systems; developing theories on social human activities, social norms and behaviours to model the Social Web involves various fields and areas, like Computer Science, Economics, Mathematics, Operational Research, Organizational studies, Philosophy, Politics, Sociology, Psychology, Systems Theory etc. [70], well known already to the area of HumanComputer Interaction ( $\mathrm{HCl})$. Especially Ambient Assisted Living (AAL)], a subcategory of $\mathrm{HCl}$, is focused on understanding and designing technology in the context of purposeful, meaningful activities [61]. In the scope of AAL, N. D. Rodríguez et al. [62] have ranked existing ontologies for recognising and modelling human behaviour to facilitate the development of artificial logic: Composite Capability/Preference Profiles (CC/PP) [40], SOUPA [17], W3C Delivery Context Ontology (Site 25), Context Ontology (CONON) [68], Situation Ont. [74], Context Broker Architecture Ontology (CoBrA-Ont) [18], Context-Driven Adaptation of Mobile Services Ontology (CoDAMoS) [58], ontology for Sensors in Palm computers (PalSPOT) [59], [60], Context Ontology for Mobile Environments (mlO!) [56] and Pervasive Information Visualisation Ontology (PiVon) [28]. They documented analytically what concepts each ontology supports, 
e.g. social interactions, devices, environment, location etc., including some behavioural aspects, like behaviour granularity or behavioural models, where PiVon gets the highest score. It is also important their qualitative analysis that $\mathrm{mIO}$ ! stresses its modelling on device interaction, PalSPOT on the user activity in the environment and with others, CONON on activity planning and services, PiVOn on location and device-based services, and Situation Ontology on temporal context operations. CC/PP enhances device and network capabilities, while CoBrA elaborates on special data. CoDAMoS brings out roles and (hardware and software) services; Delivery Context treats (hardware and software) interfaces and networks; and SOUPA, time, locations, and policies.

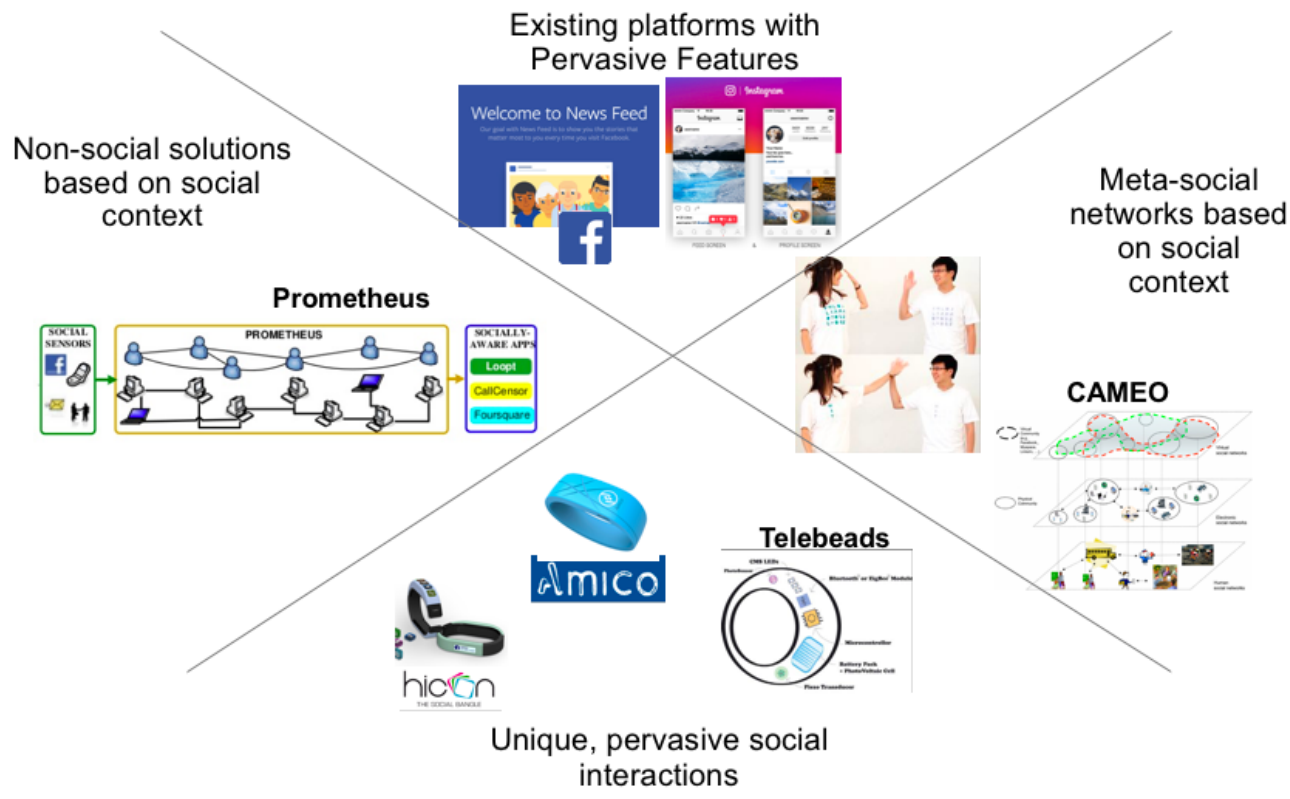

Figure 5: The different areas of research for pervasive social networking

Despite the reusable work of prioritising pervasive ontologies for integrating data coming from sensors, the work of Rodríguez N. D. et al [62] puts less emphasis on the behavioural aspect of human activities and on modelling activities from social networking. Some of the available theories that may be used to understand better modelling Pervasive Social Networking are out of the scope of social networking, like the theory of Connectivism [65] that integrates principles from chaos, networks, complexity and self-organization to explain how people learn through technology in the age of mass information. Under the umbrella of AAL, Affordance Theory [47] may also be found, which emphasises on the proper design of interfaces for objects to guide users on the proper usage of an object, as well as Activity Theory [20], [22], [35], [53] that studies the evolution of human behaviour, social knowledge, social norms and learning procedures through time; while there is also work that tries to bring together Affordance Theory with Activity Theory to exploit the expressiveness of the latter [37]. In the direction of modelling Pervasive Social Networking, Activity Theory seems promising on modelling social networking applications, while it is already the basis for modelling ActivityStreams. The main concepts of Activity Theory [35] that make it distinctive from other theories are: (a) the construction of a human activity as a set of subject, object, and tools (i.e. instruments) that are used to generate an activity outcome, under certain social norms, performed in the context of a community, and affected by rules imposed by division of labour; (b) the activity hierarchy, where activities are on top of human behaviour triggered from motives, then they resolve into actions guided from goals and sub-goals, and then actions end up to operations that actors complete subconsciously; (c) the learning procedure that takes place through time for an actor, defined as internalisation when the actor learns an activity, which evolves into an action and then into an operation (e.g. a driver does not have to think how she changes gears after some months of driving), and as externalization when she unlearns (e.g. a tourist in UK has to her driving style); (d) the instrument or tool used during an activity integrates social knowledge through ages.

All these principles on top of which Activity Theory is constructed incorporate years or research from anthropology and sociology, and make Activity Theory an ideal framework for modelling content and context under pervasive social networking. In detail, the analysis of a technology in a user-centric way is semantically rich under the Activity Theory and may categorise technologies and devices based on the activity hierarchy, thus how and when they are used (i.e. see in Figure 6 an analysis of the social media landscape under Activity Theory). Indeed, a relative analysis of contextual modelling under the Activity Theory has already been done in research [32], nevertheless it puts emphasis on the components of the activity and not on the activity hierarchy, structure and evolution. Moreover, Karanasios S. et al. [36] introduced AMOn, based on Activity Theory, as a higher ontology to link it with a layer of communication ontology (e.g. hand gestures, questions, language etc.), and then with specific dictionaries (e.g. WordNet) under a multi-layer methodology to facilitate the integration and extendibility of the Activity Theory with existing ontologies [1], [52]. This three-layer structure makes AMOn, and Activity Theory in the broader sense, appropriate for combining ontologies from different domains that interrelate under Pervasive and Social Networking too. 


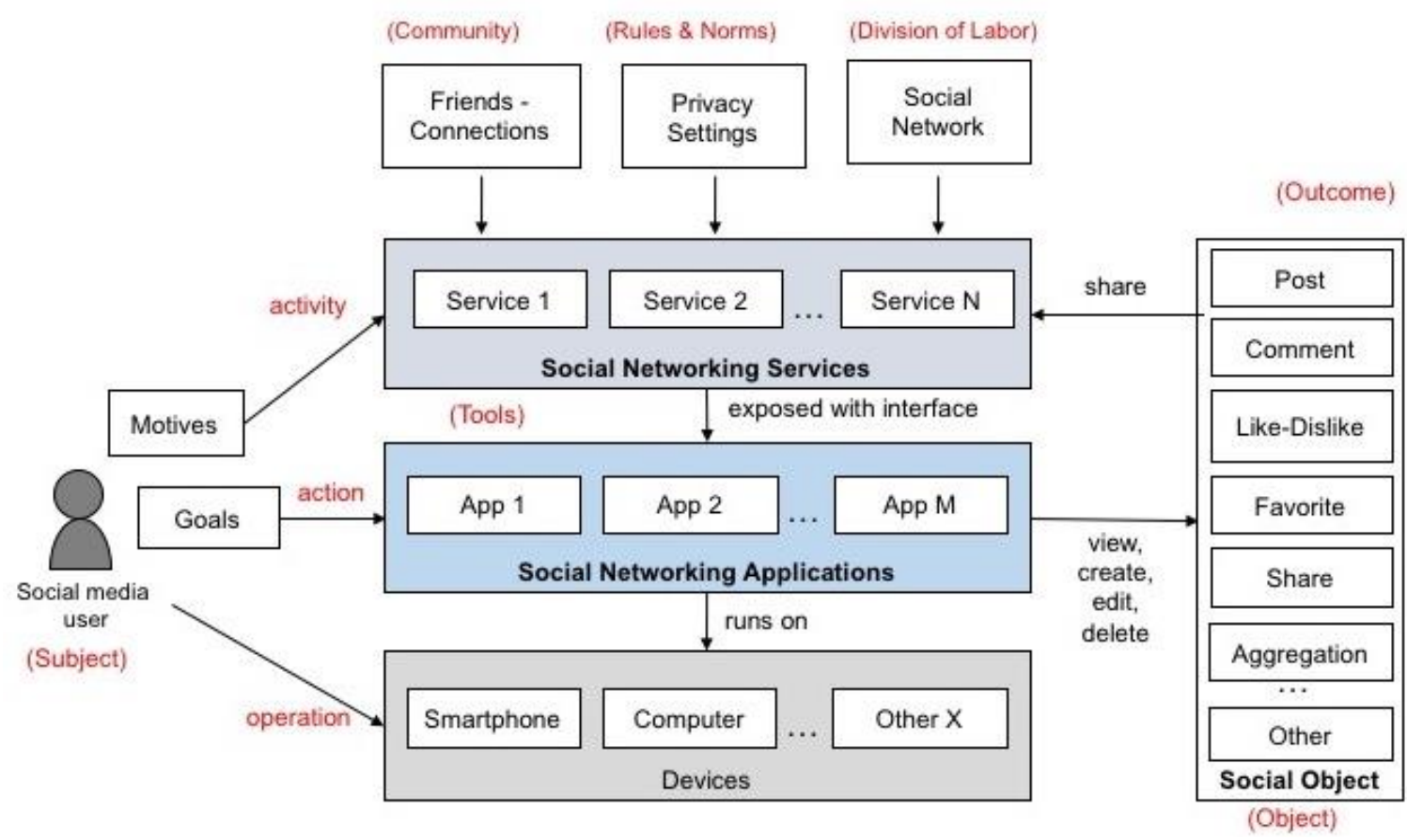

Figure 6: Modelling the social media ecosystem under activity theory

Overall, contextual data in the notion of pervasive computing improve personalised services, while activity-based data may offer hyper-personalised user experiences. Thus, it becomes clearer why tracking a human activity in the form of Activity Theory and studying its evolution through time may improve the modelling of pervasive social networking, and may offer strong incentives for business exploitation through personalisation and hyper-personalisation. Towards human activity reasoning, numerous approaches have been proposed [64] without providing tangible results and clearly described models; but before improving such forms of automation, it should be agreed what an automated service should be like in pervasive social networking.

\subsection{Social Agents}

Pervasive Social Networking requires establishing social connections ubiquitously, thus taking proper decisions on initiating a communication with other services. The implied agency of a solution acting as a representative of the user on social networking tasks requires prior knowledge of the environment and understanding of user's behaviour, but also a set of social rules and goals according to which an agent would initiate social interactions. To achieve such automated or semi-automated social interactions, social agents have already been introduced in research, grouped in three main relative categories (Figure 7) (a) social agents to change users' behaviour, (b) social agents to produce content and create connections over existing social networks, and (c) social agents that show characteristics of social intelligence when interacting with users.

On the basis of behavioural intervention, a social agent is described as an artificial agent with two dimensions: processing capabilities and differentiated knowledge of the self, task domain, and environment that drive to the development of a social behaviour; every social agent may be positioned in a two-axis area, based on its processing capabilities and its differentiated knowledge of the world [12]. On the same track of developing social behaviours, a social agent [14] (a) should act for the good of the owner either to prevent (i.e. suppressive) or to encourage (i.e. coaching) a behaviour, while (b) the collaboration with other agents requires agreement and compliance with common rules and beliefs among individuals, with (c) limitations and dynamic behaviours to emerge during networking of the agents; in other words, a social agent should belong in a network of agents in order to align its intervening behaviour with the social norms and rules where its owner lives under. Indeed, there is applied research on social agents that affected positively users' behaviour on ecological issues [63], as well as simulations on how social agents may form symbiotic and competitive connections in social networks [26]. 


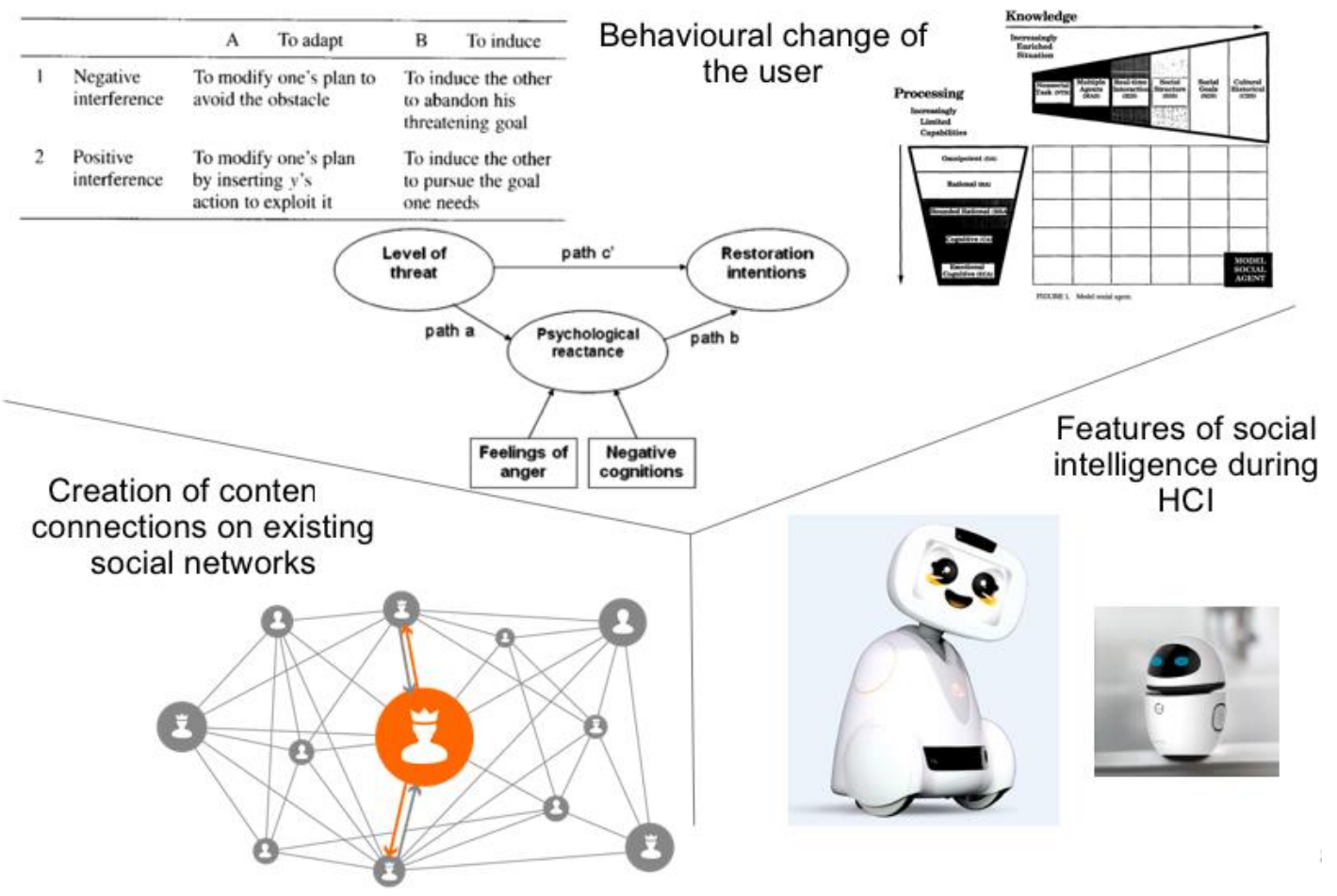

Figure 7: The three main areas of research for Social Agents

In practice, on existing social media platforms, social agents may act either to generate more intriguing content or improve social connections in the network: (a) There are agents that try to improve their position into a network of agents, e.g. by creating a bridge between two clusters or by establishing a connection with the most important node; however, such goals suggest previous mapping of the network, and may drive agents into competitive behaviours [66]. (b) There other social agents that create interesting content automatically and connect users through it, by using decision systems to resolve user preferences and user context [25]. (c) Other agents may attach social context on physical objects, generated from users' social media profiles, to form a network of Social Internet of Things (SloT) where users may establish indirect social connections through the objects, in a basic level of agency [4], [5]. (d) On an opposite direction, physical objects may act as content generators by making their properties available to other applications and sharing their output on social media [41].

From the perspective of social intelligence, research focuses on boosting users' engagement during their interactions with agents. In that direction, agents are characterised as social because they collaborate with other agents to achieve their goal, yet they may be separated from the ones that show social skills while interacting with users [24]; the latter group may be organised in the following categories: (a) socially evocative, humanised robots to increase user acceptance, (b) social interfaces to run social operations instead of humans, (c) socially receptive to learn through the interactions with humans, (d) sociable to initiate interactions with humans in order to achieve their goal, (e) socially embedded to respond to the social group where they are placed, (f) socially intelligent to simulate human social behaviours. In that direction, it has been found that the physical interface of the agent, including hardware, may affect the quality of the interactions [45]; the results have shown that the external appearance of agents affects such interactions, repeated and incrementally improved behaviours increase users' interest on agents, as well as agents that show emotions, affection, and memory of previous interactions.

Overall, in a level of abstraction and general principles, a social agent in social networking may have knowledge of its environment and different processing capabilities, while it is connected with other agents in order to improve its performance and establish social connections among users. Thus, social agents should not be judged based on their processing capabilities, or on showing social skills while interacting with people, but on their effectiveness on establishing social connections with other people.

\subsection{Summary and Gaps Identified}

Nowadays, social media endeavours are hindered by community-related impediments, as building a community from scratch usually fails (i.e. even if it manages to attract publicity like Diaspora) due to the prevalence of Facebook, Twitter, Instagram and other well-established social media platforms. The alternative option of leveraging existing social media communities through a meta-platform faces the restrictions imposed by the relevant API policies. Despite progress on alternative social networking architectures to provide users with control of their data, such approaches have not been widely adopted by users as (figure 8): (a) they introduce more complexity and high costs without clear added value to 
the users, (b) users typically show little interest in solely privacy-aware solutions, without any distinctive novel offerings, (c) users prefer using a new social media application to create unique content from moving their existing social connections to a decentralised solution, which also is not yet clear how it will be economically viable.

Thus, the main research challenge is how some technology acting pervasively on social networking tasks may become acceptable from users who seem to be quite sensitive on the quality of their social interactions; or in other words, what the privacy rules and social features should be in order to balance pervasiveness with users' comfort and joy. However, there is no clear methodology on developing social features for new platforms, while researchers and entrepreneurs are based on their epiphany and intuitiveness. Additionally, there is no agreement on evaluating a new social networking design; while some researchers emphasise on privacy features, others focus on technical independency, others on simulation practices, or coming up with genuine features.

The main practical challenge is modelling the Social Web towards a standardisation path, without considering social interactions as a topic of social networking only, but as a valuable source of context that can boost multiple areas of computing, including pervasive interactions of users with different systems and platforms. Pragmatically, the standardization of the Social Web is not yet ready to capture the changes in users' behaviour, as contextual models are not integrated in one social design and are not driven by proven theories. The main problem with existing schemas is that they either focus on user-generated content (e.g. ActivityStreams, SIOC project) or on users' actions (e.g. PiVon, $\mathrm{mIO}$ !, ConOnto, CoDAMoS), and not human activities with broader motives and rules that imply connections among people. Mobile devices have boosted tracking digital traces that imply such interactions even outside the Web, but pervasive computing is not yet integrated with social networking under a common model in order to actually form Pervasive Social Networking.

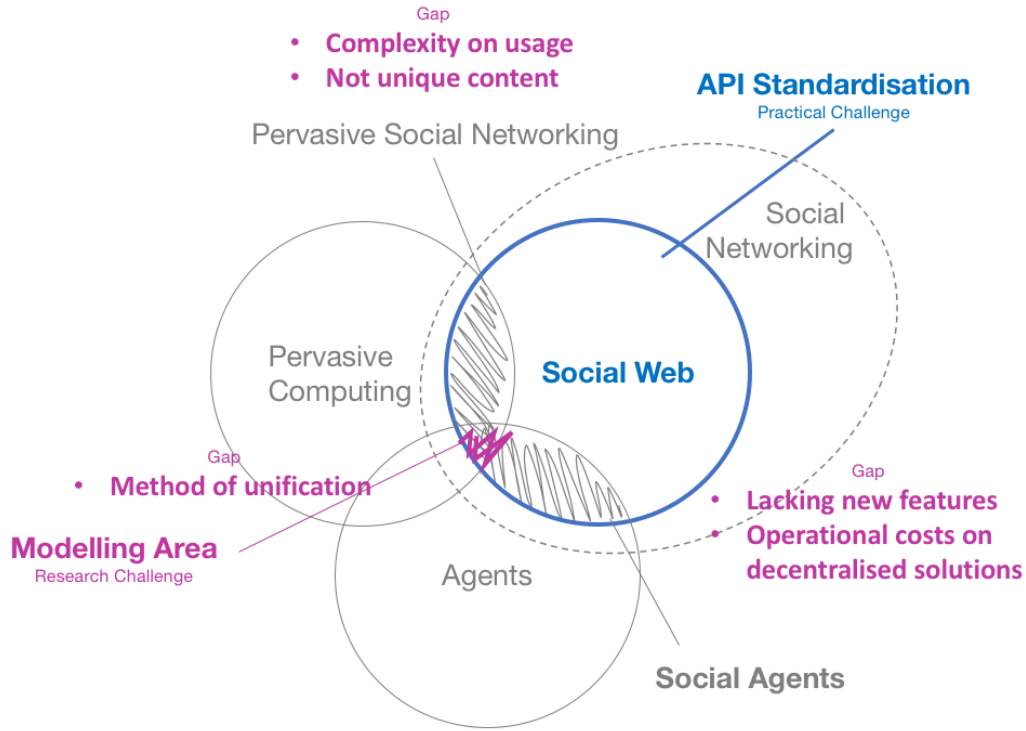

Figure 8: Gaps and challenges identified for different areas

The answer to that emerging reality may be moving towards decentralised solutions (i.e. even meta-platforms) based on existing knowledge (i.e. reusing content and connections from existing social media platforms), with strong personalisation features and high level of pervasiveness to reduce the user effort. A social agent with strong pervasive characteristics, proper rules and acceptable social behaviours may be the solution, and a relative design to that direction is presented in detail in the following chapter.

\section{A Social Agent for Pervasive Social Networking and Personalised Services}

In a highly competitive digital environment, people should use pervasive services to reduce time spent on consuming content and effort needed while interacting with technology. Such services may be responsible for tracking personal activities, understanding activity evolution over time, predicting possible upcoming user's actions, and interacting with user's physical environment in a meaningful, trustful and interoperable way. Then, users may improve their lifestyle through tracking their activities, monitoring progress in new skills, interacting with interesting people in proximity to create new social connections or with devices and services in proximity to improve pervasive interactions.

The concept addressing the relative research questions (see Table 1) is called Social Agent and it emphasises on characteristics and principles that such an entity should support. In the following paragraphs, initially the broader area of Pervasive Social Networking is framed under the value chain of pervasive data, and then the generic concept of a 
Social Agent is presented in detail. Finally, a technical solution that may implement a specific Social Agent is modelled, by developing a methodology to map and exchange data from different resources under the umbrella of Activity Theory.

\subsection{The Value Chain of Pervasive Social Networking}

To put the landscape of Pervasive Social Networking into perspective, as analysed in section 2, the usage of the Value Chain [55] is used as a tool to understand the importance of information and the value it generates. As seen in Figure 9, the basic processes related to pervasive data are the following:

- Collecting Data from various resources is required to reuse them for social networking purposes or modelling social context; data from social media, sensors that imply activities, mobile and wearable devices that provide user-specific measurements, objects with loT-capabilities, information from open Web, even dedicated applications to collect information or manual input from users may be used.

- $\quad$ Mapping Data under a common model is essential for an interoperable solution; through proper transformation to match a schema, data curation to improve quality of data (i.e. especially sensor data), feedback collected from users to validate curated input, mixing of primarily and secondary data to define an activity, and then map them into a proper schema.

- Analysing Data collected through time may generate knowledge and allow a system to make decisions pervasively; through network analysis on communities, signal analysis on secondary data, statistical analysis, predictive analysis or pattern recognition, in order to extract knowledge from the collected data. This step may also include applying rules and knowledge from behavioural theories.

- Sharing Data among applications is the main goal of digital social networking to create a social object and connect people with each other; sharing instances of data objects, statistics on the user's activities, extracted topics of interest, or just a user profile are some of the forms that such messages may have.

- $\quad$ Creating (social) Connections among users may be established with direct (i.e. friendships) or indirect ways (i.e. interact on a social object). Those interactions may be explicitly triggered by users, engaged through software based on contextual information or based on the outcome of some analysis (e.g. the social proximity between two users). This must be the final, ultimate goal of pervasive social networking, and it has strong dependencies with previous steps.

Throughout these steps (1-5), various design and technical parallel decisions should be taken into consideration. Initially, (6) how the community should be built and grow, as a new social network, as a mix of new connections based on existing communities, or as temporal connections; (7) what tools users may use to connect with each other, as the channel of delivery may be an application, an agent acting as representative of the user, mobile devices or dedicated wearable ones to trigger a social interaction; (8) how services should be exposed, through the Internet, through direct connections engaged from users based on a signal (e.g. notification), or through close-distance protocols that imply trust (e.g. NFC); (9) the architecture of the network and where users' data should be stored, in centralised, decentralised, distributed or hybrid architectures; (10) the security over these sensitive data and interactions, with strong needs on encryption, sophisticated authorization and authentication, and proper verification on users' identities; finally, (11) the privacy rules and settings under which data are shared and exposed to other users, on data types that should be controlled by privacy settings, on the connections that should be allowed or blocked, for limited visibility on specific instances, on notifications, and for changes in system behaviour based on specific context.

\subsection{Defining a Social Agent}

A Social Agent (figure 10) is a technology that tracks digital traces of its owner, models them properly as human activities and makes them available as abstractions to other Social Agents in proximity (e.g. location, social, interests etc.) to create pervasive social connections based on the privacy settings of its owner. Tracked data must be stored in a trusted repository, centrally or locally, and may be subject of further analysis to create meaning and value for the owner of the agent. These data may come from various resources, as input from (a) sensors, (b) wearable devices, (c) cloud services, (d) direct input from the owner, (e) user's feedback on agent's output or (f) from interactions with other Social Agents. That information must be stored and modelled under Activity Theory to enable standardised processing and interoperable communication with other systems. Then the Social Agent may be able to use any contextual input (e.g. date time, current user location) to estimate either the currently undertaken activity or a prominent set of activity types that the owner is about to undertake, and use that output in the following ways: as information shared with other Social Agents in the network to create pervasive connections with them in proximity (e.g. they may be interested in the same topic, or undertake similar activities); as input to cloud-based services to enable pervasiveness (e.g. if the user is about to run, she may ask for weather forecasting services); as output presented to its owner in order to use it as training feedback and improve future predictions. A more compact and generic definition of a Social Agent is the following: 
Definition: A Social Agent is a set of micro-services to store and analyse digital traces of human activities for further social networking purposes, running over some private hardware (i.e. virtualised or not, even on the cloud), exposing multiple pervasive interfaces with other Social Agents (in proximity or not), with its owner's trusted devices and with external personalised services based on standardised, semantically rich activity-based messages.

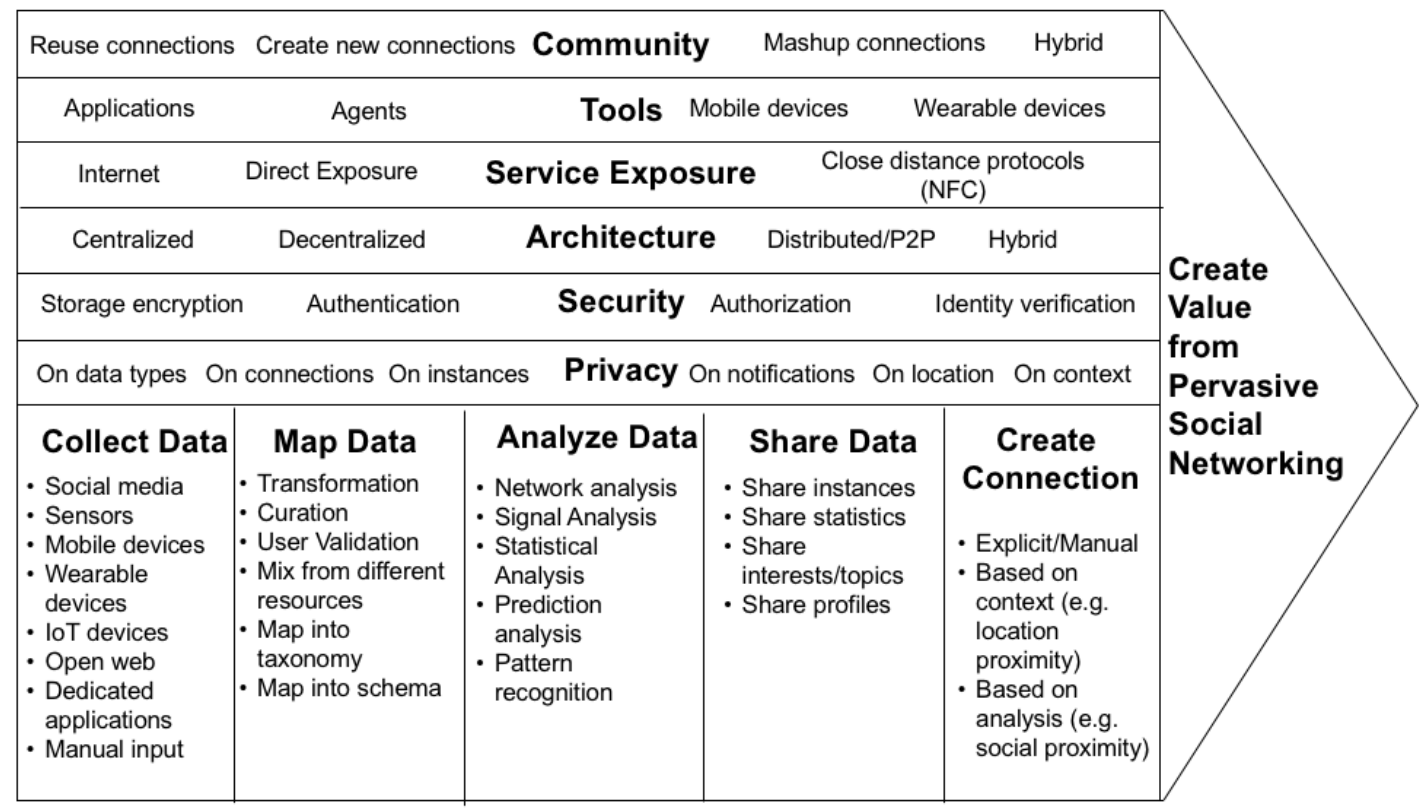

Figure 9: The information value chain of pervasive social networking, based on the work of [55]

The concept of a Social Agent should be focused on achieving the goals of its owner during social networking. In each case, transparency, integrity and trust are key values that a provider of a Social Agent should offer to the owner of the agent. In an essence, a Social Agent describes a transformation in the way social networks work nowadays, from systems that are based on predefined connections with people, using connections explicitly defined by the users or created through content shared among users, to a social connection that may engage the opportunities of interactions in physical space. A Social Agent may not be responsible for keeping people connected from distance, as such scenarios lack pervasiveness and require explicit input from users (i.e. such features may be implemented in a mature solution), but should specialise in connecting people in physical proximity. In other words, the envisioned Social Agent may not subsidize existing social networking platforms and it should act supplementary to them to offer to users social networking in close distance.

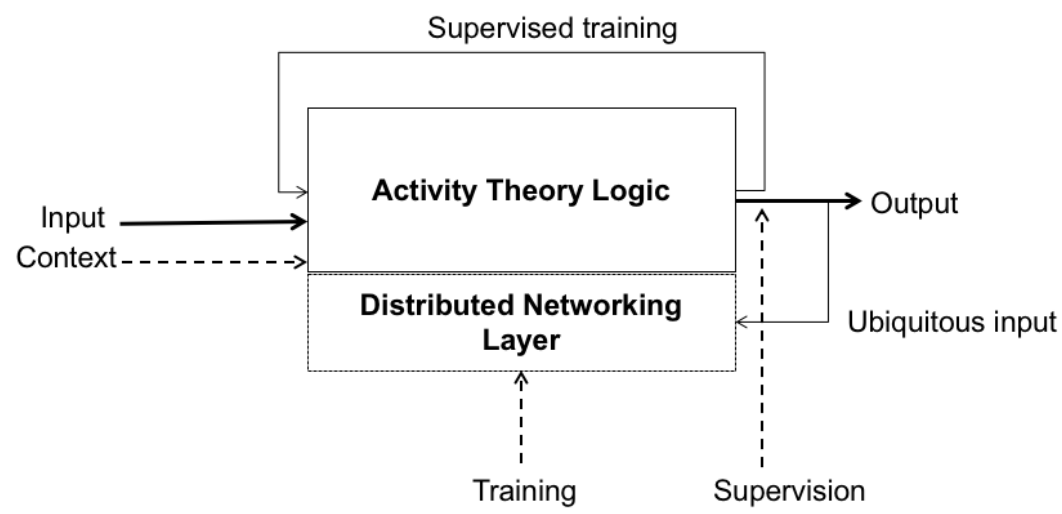

Figure 10: A social agent to track, analyse and share personal activities

As a Social Agent should be user-centric - meaning it should not depend on accessing other users' data in order to establish a social connection - while it should be standardised and extensible to avoid the need of centralised platforms to fulfil its goals. Additionally, the Social Agent should support personalised services to minimise the required user input, therefore support its pervasive character and increase its chances for social adoption. In detail, the main characteristics of a Social Agent should be:

1. User-centric: The owner should control any data stored in her Social Agent, generated through either interacting with other Agents, devices or syncing with cloud-based services. Additionally, data collected with 
a Social Agent should be related with the owner and stored in the form of human activities. Thus, any contextual data should be stored under an activity entry of the user. Any additional data that have not been produced by the owner, or the owner has not interacted with, should not be stored in the Social Agent, e.g. the activities of other users or media content that the owner has not produced or viewed yet.

2. Pervasive: The functionalities of the Social Agent should be pervasive to the extent that the privacy of the owner is not violated. Pervasiveness should apply both to the social interactions with other Social Agents and to the interaction with other services that may improve owner's experience, e.g. ask for weather forecasting or traffic information services based on the upcoming activity.

3. Standardised: The way data are stored and shared as messages with other Social Agents and other services should be standardised. Activity Theory should be the umbrella theory under which data are modelled and exchanged, while any case-specific schemas (e.g. pervasive schemas) should extend data structures of an activity entry. Otherwise, the Social Agent cannot be independent of centralised platforms, and owners will never have full control of their data.

4. Extensible: A Social Agent has been intentionally described in a generic form, where specific social features and functionalities are not defined; each implementation of a Social Agent may release a list of available functionalities relevant to the social and business context were deployed. If stored data are standardised, different functions over different constructs of an activity entry may be applied, to exchange proper messages with other Social Agents or services, e.g. presenting the frequency of an activity type, a list of the most popular activity types, or comparing the duration of common activity types between two users. A social agent must support at least a minimum set of functionalities in each release.

5. Hyper-personalised: A Social Agent manages data that describe the behaviour of its owner. Thus, while communicating with external services, it should focus on connecting with interfaces and obtaining results that are related to the owner's interests and current context. Otherwise pervasiveness will not be relative, and more input by the owner will be required to perform an interaction with the Social Agent.

6. Networked: A Social Agent should be characterised as social only if it is connected with other agents to complete a social goal for its owner. Otherwise it is just a personal agent. The option for owners to disconnect should be given. Nevertheless, they should be also encouraged and facilitated from the Agents to connect with other people or agents in proximity.

To realise these six characteristics, and in extension the concept of the Social Agent, there is a set of suggested functionalities that a Social Agent should be able to offer:

- $\quad$ Track a human activity both in physical and digital world, via direct (from the user) or contextual input.

- Model properly and identify activity internalisation and externalisation, based on the Activity Theory hierarchy.

- Integrate social rules and dynamics (i.e. labour on Activity Theory) on predicting activities, aka the social context where it operates.

- Generate contextual output as input to other pervasive systems or Social Agents, to enable pervasive computing and socialising.

- Switch to the desired mode based on the occasion, e.g. a business social agent versus a sports/training social agent.

- Store data either on devices locally or centrally on a cloud storage, private, corporate or public one, based on the predefined user settings.

- Help users to adapt in a digital world, by informing the owner about possible interactions with other agents, services or devices in proximity, in order to encourage or discourage interactions.

A social agent should be extensible for and adaptable to its owner's needs and environment through training. It should collect and store such knowledge over training sessions. Relatively to the training capabilities, a social agent should be able to (Figure 10):

- Ask periodically the owner for validating an entry with her feedback on what activity is actually performed, especially when contextual input implies a new type of activity.

- Be trained with predefined packages, to adapt in social norms and gain knowledge on specialised topics. 
- $\quad$ Learn through networking. Thus, owners should learn from people they interact with, or through similar users in the network, giving to the owner the option to learn by others. Proper privacy and anonymisation layers should be configured.

Moreover, the pervasive privacy settings of a Social Agent are important for establishing trust with users and implement pervasive social interactions effectively, without going through complex privacy settings (e.g. the Facebook privacy settings), under the following privacy settings:

- Availability-mode settings may allow owners to define the group of people they want to interact with. The suggested options may be (a) to allow public interactions with any Social Agent user that is in proximity, or (b) to become visible only to friends that are close. Of course, an owner must be allowed to (c) switch completely off discoverability of her agent.

- $\quad$ Proximity settings may allow users to enable pervasive social interactions for different proximity distance with other users. For example, settings based on physical distance may define user's areas of trust [34] as (a) intimate space if two users are closer than $0.5 \mathrm{~m}$, (b) personal space below $1.2 \mathrm{~m}$, (c) social space until $3.5 \mathrm{~m}$ and (d) public space closer than $7.5 \mathrm{~m}$; beyond that distance it is considered that users would not be interested in pervasive social interactions, rather than traditional social networking.

- Location settings may allow users to define areas or places where the device should change mode automatically. For example, in a working establishment a user may choose to share only business activity types, while inside her home every type of interactions or full access on data is allowed.

Apart from the social-agent-specific settings, it is expected that a Social Agent supports common privacy settings, like defining trusted connections and groups of people, defining trusted devices, defining personal devices and defining specific visibility options on specific entries or activity types.

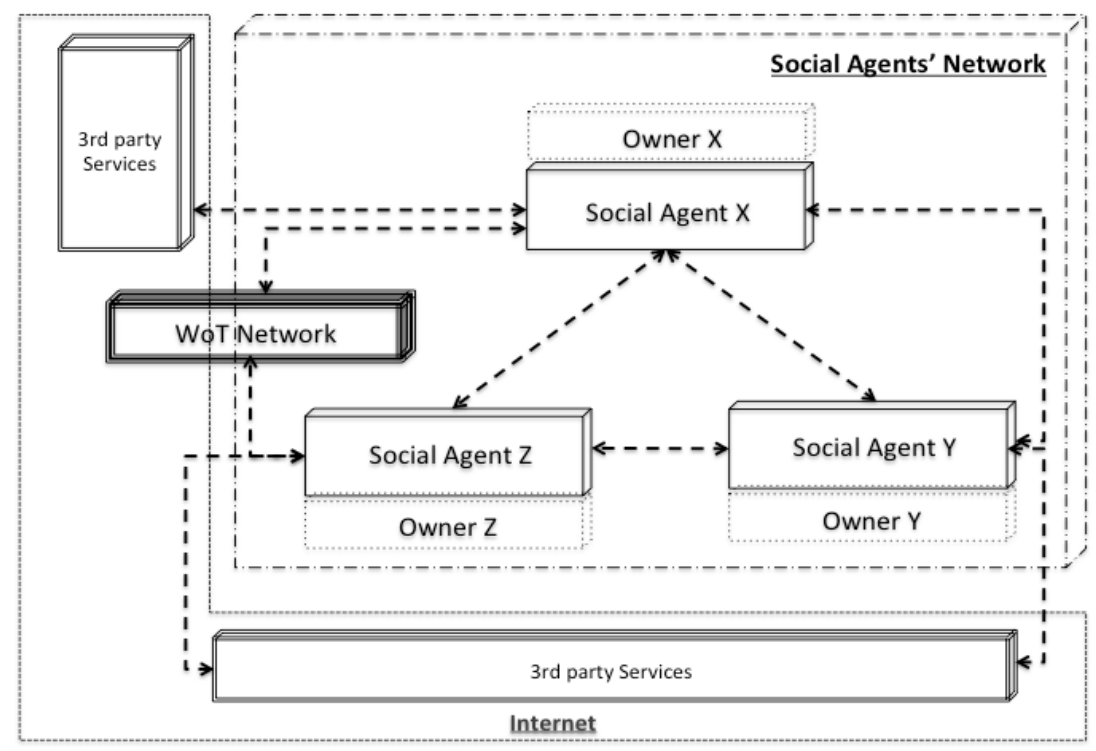

Figure 11: An inter-connected social agent that allows communication with multiple entities

A Social Agent should form social networks pervasively to exchange knowledge and reuse services across the members of its social network (see figure 11). In that direction, physical distance is one of the most important privacy dimension of pervasive social networking, as it may be used for choosing the proper protocol to establish technical networks and change agent's behaviour respectively. In more detail, Social Agents may interact over existing networking infrastructure - like the Internet - or through close-distance protocols like Bluetooth or NFC, they may interact with objects around them connected with the Internet of Things (loT) exposed through APIs on the Web as well (Web of Things - WoT), they may exchange content with third-party services on the Cloud, or they may synchronise owners' data with central personal repositories. Such connections may be developed pervasively, but the owners should be allowed to trust certain connections to form at the end an actual pervasive network of permanent (or descriptive) and temporal (or pervasive) social connections.

In practice, as mentioned already, agency in social networking requires strong pervasive characteristics. For that reason, as seen in Figure 12, a Social Agent may collect various types of information to construct a user activity from a set of different digital traces. That information may be: (a) Possible user's input on what is her current activity; (b) A set of contextual data, like location, device characteristics, or social context for users identified in physical proximity; (c) User settings on what is the current mode of the user and how she expects the agent to behave or user input on 
what is the goal of the undertaken activity; (d) The outcome of the activity, either measured explicitly based on the activity type or collected in the background based on common measurable metrics (e.g. distance, time, score). Given that information, a social agent should be able to identify the current activity type (i.e. if not given by the user), the hierarchy of the activity, as well as possible upcoming ones based on user's history and pattern of behaviour. This set of possible activity types, may be used both to establish a pervasive connection with a user in proximity, and to interact with a cloud service or a device in proximity in order to increase user's benefits through pervasive computing and personalised services.

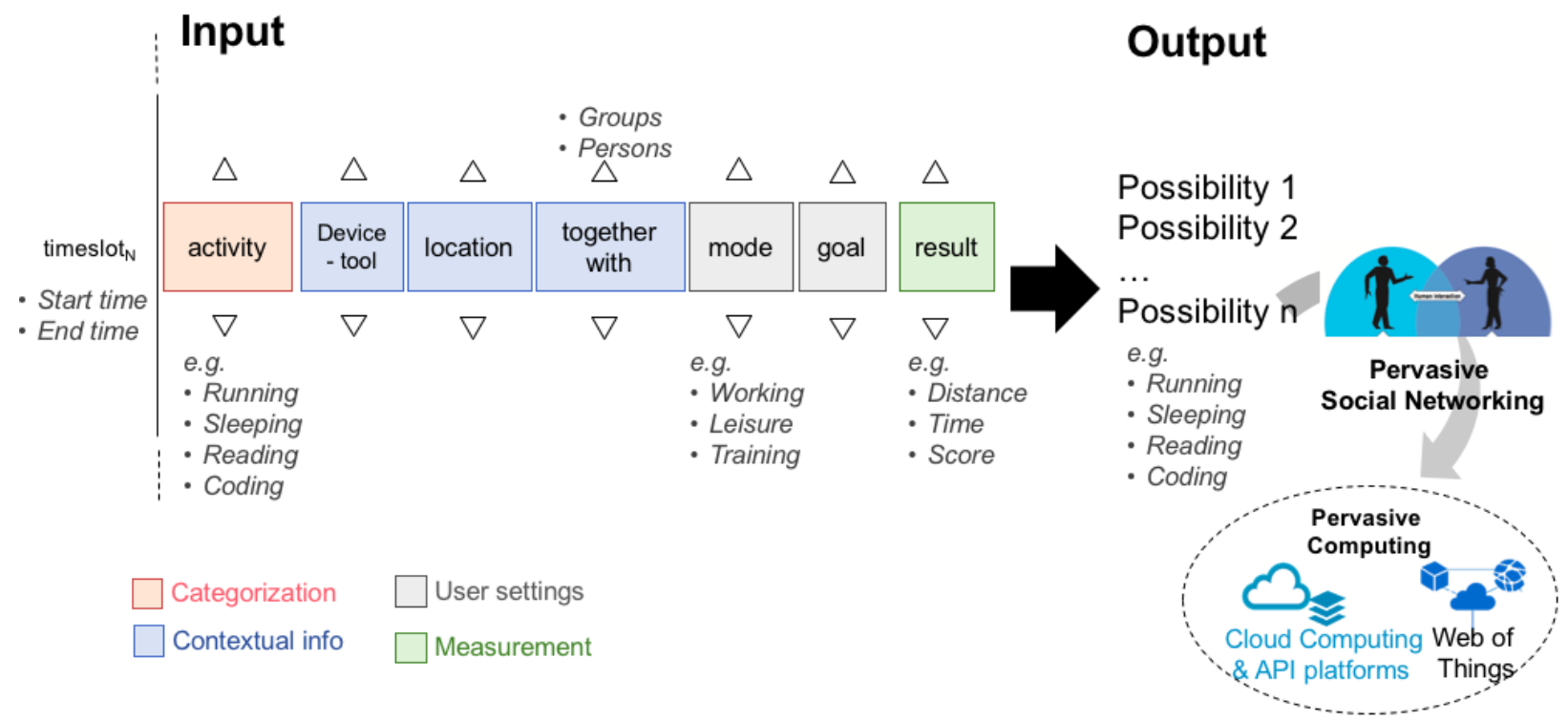

Figure 12: A social agent connecting its output with new services to expand its capabilities

Overall, Social Agents should represent their owners, act pervasively to protect and help them on their daily activities, enable trustful handling of personal data, enable social networking and commercial services to be based on open standards, decentralised solutions and ubiquitous services, and spread value equally among users and organisations acting as a meta-platform, without considering centralised platforms as monopolies that manage information flow. Furthermore, users should be in the centre of the design, existing social media platforms should be part of the design to reduce required input and reuse available content, and e-businesses should build new-age, personalised and hyperpersonalised pervasive services over business platforms that act as middleware.

\subsection{Modelling a Social Agent}

The introduced Social Agent covers the main processes in the value chain of pervasive social networking, as presented in figure 9. Its suggested logical components, as seen in Figure 13, are:

a) Activity Input: A Social Agent may receive input (i) from dedicated applications that ask an owner for her daily routines and their undertaking activities, (ii) from cloud-based services (CBS) that offer APIs with activities that a user has completed in other applications (e.g. exercising in RunKeeper, or socializing in Facebook), (iii) from loT devices to describe the context and the environment of the human activity, and (iv) from sensors on mobile/wearable devices to give specific measurements on outcomes, duration and activity context (e.g. heart rate, pace rate etc.).

b) Networking Input: Carefully developed services may insert sets of activities that change the behaviour of the Social Agent, especially in cases that it is desirable to change the outcome and affect the interaction with a service or change owner's behaviour [13]. Additionally, this interface may be used to add rules to the agent and specialise it in specific practical domains (e.g. using an agent during exercising or setting new diet rules for the user).

C) Activity Handling: A Social Agent should handle data in a common way and should model them as human activities (i.e. under the Activity Theory), under a standardised and extensible schema. Proper mapping among data inputs with the schema is required, as well as the logic to generate outcomes over those data. For example, activity internalisation realisation, mapping routines to activities, cloud-services data to actions and sensor data to operations.

d) Knowledge Expansion: A standardised Social Agent may be expanded with pervasive or other ontologies to enable knowledge on new domains, and dictionaries to standardise the way human activities are described. 
e) Activity Output: A Social Agent should expose only abstractions of data as output to services, like statistical analyses on different dimensions of the human activity modelling or techniques to predict the upcoming activity types of the owner given the current user context. There is no reason to expose raw activity entries to another service, not even to synchronise with an external service, as an agent should remain user-centric.

f) Social Interactions: A Social Agent should generate social connections among users based on carefully selected social features. These features should be predefined ways for agents to connect users over their human activities, like comparing two users' activities or finding common topics. A Social Agent should expose its presence to other Social Agents or other pervasive services in proximity through close-distance networking services, like Bluetooth or NFC, and it may change its social features based on the type of networking connection, for example enabling the comparison of activities only over a Bluetooth connection.

g) Settings: The user and owner of the Social Agent should be able to control the authorised services, edit her profile and visibility on them, control the connections with other users if permanent connections are enabled through the offered social features, otherwise see a history of the interactions and manage it appropriately, and manage the permissions on devices, social interactions, services and users on accessing personal information.

h) Pervasive Social Capabilities: The user should be able to interact either with other users through her Social Agents or to share information with pervasive services to improve her user experience.

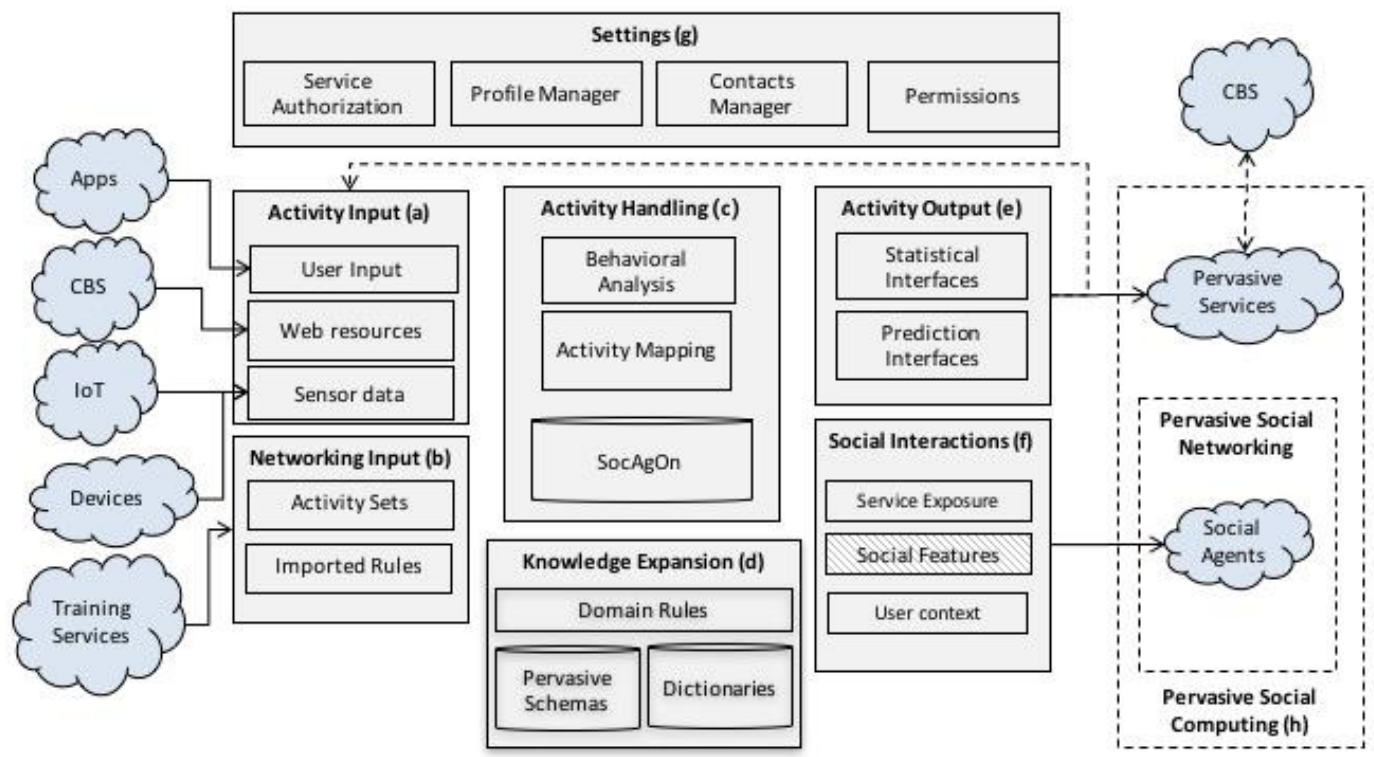

Figure 13: A general architecture for a pervasive social agent

The described functionalities of the Social Agent have been modelled under an ontology named SocAgOn (Site 13). Modelling a Social Agent under the umbrella of Activity Theory facilitates the integration of existing schemas and vocabularies from social media and pervasive computing, to extend the knowledge capabilities of an agent. The challenge was to extend the SocAgOn schema with knowledge from different domains, to facilitate reusability and extendibility with data from different resources. The selected approach to integrating different schemas is the threelayered methodology [37], as it creates less dependencies on connected schemas and makes the final schema easily reusable and extendable. As seen in Figure 9, the area of Pervasive Social Networking modelled under SocAgOn may be extended with schemas on social media and pervasive computing using the following structure:

- $\quad$ On the upper abstract level, there is Activity Theory as an umbrella where each activity entry should be mapped, and AMOn [37] is the suggested schema in our modelling. Thus, every tracked digital trace should be bound under a human activity; for example, user-generated content should be attached under an action of sharing a media object (see also Figure 6).

- $\quad$ On the middle reusable level, SNP-related schemas should be integrated. ActivityStreams (Site 6) is the selected schema to describe activities on social networking applications from existing cloud-based services. SocAgOn has been used to model Pervasive Social Networking, defining the concept of a Social Agent. As both schemas are based on Activity Theory, semantically mapping similar classes was a straightforward task. 
- $\quad$ On the lower, case-specific level, each pervasive solution should choose the schema that is appropriate for the developed application. The goal of this layer is to define the contextual information of main Activity Theory objects, and to include detailed vocabularies that will describe the business case and make the application semantically rich and pleasant to users. CoDAMoS has been selected [62] as an appropriate schema to describe the context of activities and objects, while GUMO [38] may be used to describe the context of users. Additionally, our analysis has identified appropriate schemas to expand the functionalities of pervasive social networking applications: UMBEL (Site 10) for describing daily activities and (Site 7) for web-based objects.

Figure 14 depicts the three-layered approach, and the points of integration among different schemas for pervasive social networking. Applications compliant with SocAgOn may map their data over the selected schema, while existing SNPs and devices need connectors to map their APIs over the final generated data model.

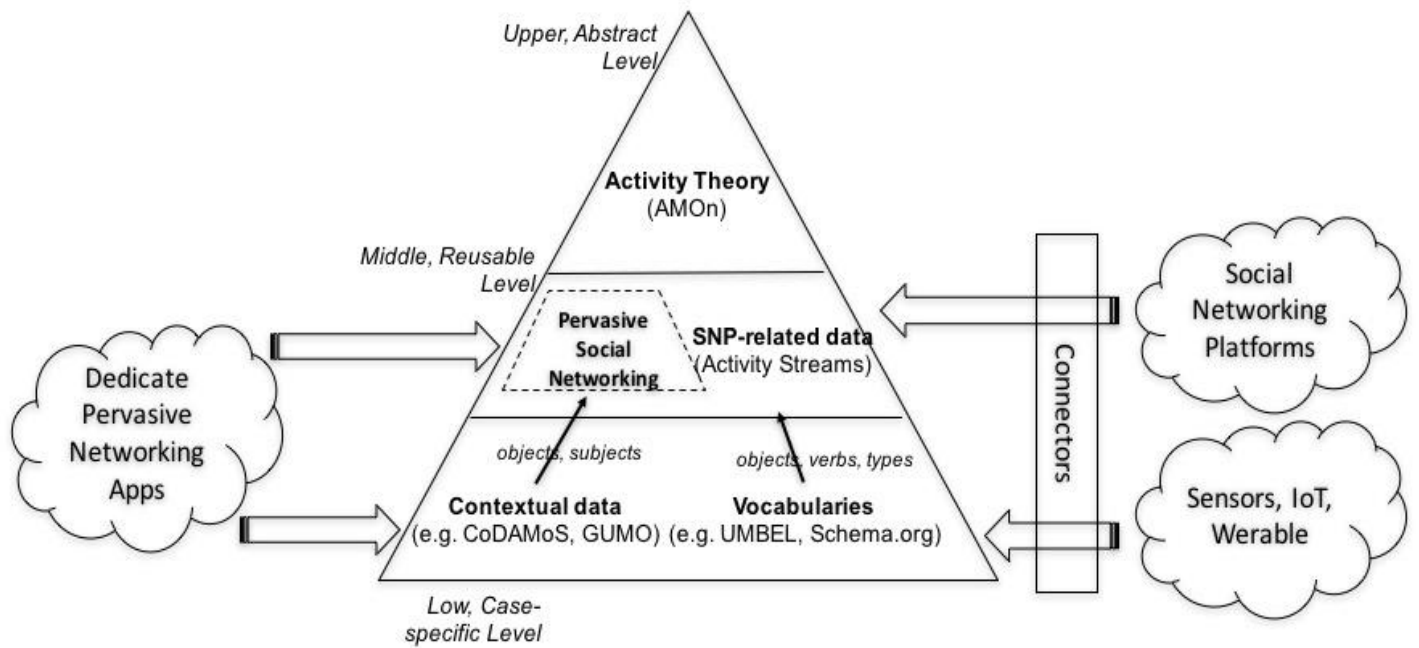

Figure 14: Multi-level modelling approach of social agents

\section{Evaluation of Work}

The evaluation of the Social Agent was concluded in two phases. In phase one, the initial hypotheses formed with the concept of a Pervasive Social Agent were evaluated quantitatively through a questionnaire based on TAM methodology, on 200 people. From the feedback collected, the concept of the Social Agent was revised as presented in section 4.1. In phase two, a feasibility analysis took place, by modelling the architecture and the schema of a pervasive social agent and by developing a prototype, as presented in section 4.2. The high-level evaluation of the concept is described in section 4.1, while the feasibility testing in section 4.2 .

\subsection{Evaluation of the High-Level Concept}

The evaluation of the high-level concept was based on the TAM methodology [44]. Initially a set of questions to test the high-level concept of the social agent was produced, and then a set of features was examined to make it clearer to interviewees how a social agent may be used in action. The outcomes were analysed statistically in the initial phase, and then the correlation among the selected criteria was investigated.

\subsubsection{High-level Evaluation Methodology}

The Technology Acceptance Model (i.e. TAM3 [71] in particular) was selected to generate the evaluation criteria and identify any correlation among different sets of them. The main challenge on designing the questionnaire was to evaluate the concept of the Social Agent without asking interviewees about technical terms, without also binding their responses to the quality of an implemented prototype with jagged features (e.g. native versus mobile application, response time, size of the community etc.). From the value chain of pervasive social networking (Figure 5), two main areas were considered important to get users' feedback: (a) the way social connections can be formed automatically in a social network when users are in close distance (i.e. the pervasive character of a Social Agent) and (b) the privacy concerns that users may have on such social functionalities. Under those two areas of interest, ten questions were generated to evaluate the concept and the characteristics of the social agent with the users (see Table 2). 
Table 2: The questions of the survey to test the concept of the social agent

\begin{tabular}{|c|c|c|}
\hline $\begin{array}{l}\text { Scope of questions asked for the underlying characteristics of a } \\
\text { Social Agent }\end{array}$ & $\begin{array}{l}\text { Referred } \\
\text { characteristics }\end{array}$ & Area of interest \\
\hline $\begin{array}{l}\text { Q1: An application to connect you with people in close distance } \\
(<50 \mathrm{~m}) \text {, based on your activities and common interests }{ }^{*}\end{array}$ & $\begin{array}{l}\text { Pervasiveness based } \\
\text { on human activities }\end{array}$ & $\begin{array}{l}\text { Social networking } \\
\text { in close distance }\end{array}$ \\
\hline $\begin{array}{l}\text { Q2: An application to collect data for your activities from the web, so } \\
\text { that it helps you understand your online behaviour }\end{array}$ & $\begin{array}{l}\text { Reusability of web } \\
\text { services }\end{array}$ & $\begin{array}{l}\text { Social networking } \\
\text { in close distance }\end{array}$ \\
\hline $\begin{array}{l}\text { Q3: An application to collect data for your daily life (through sensors), } \\
\text { so that it helps you understand your daily behaviour* }\end{array}$ & $\begin{array}{l}\text { Reusability of digital } \\
\text { traces from devices }\end{array}$ & $\begin{array}{l}\text { Social networking } \\
\text { in close distance }\end{array}$ \\
\hline $\begin{array}{l}\text { Q4: An application to understand when you improve and when not } \\
\text { in your activities* }\end{array}$ & $\begin{array}{l}\text { Agency based on } \\
\text { Activity Theory }\end{array}$ & $\begin{array}{l}\text { Social networking } \\
\text { in close distance }\end{array}$ \\
\hline $\begin{array}{l}\text { Q5: An application to predict your upcoming activities and notify you } \\
\text { for relative and useful information* }\end{array}$ & $\begin{array}{l}\text { Agency to improve } \\
\text { user's behaviour }\end{array}$ & $\begin{array}{l}\text { Social networking } \\
\text { in close distance }\end{array}$ \\
\hline $\begin{array}{l}\text { Q6: An application to inform you about devices and people in } \\
\text { proximity, so that you interact with them* }\end{array}$ & $\begin{array}{l}\text { Network of Social } \\
\text { Agents }\end{array}$ & $\begin{array}{l}\text { Social networking } \\
\text { in close distance }\end{array}$ \\
\hline $\begin{array}{l}\text { Q7: You may choose to interact automatically with people in close } \\
\text { distance, with the following settings: (a) public interaction, (b) private } \\
\text { interaction with people in your agenda, (c) only for specific groups of } \\
\text { people you choose }\end{array}$ & $\begin{array}{l}\text { Pervasiveness based } \\
\text { on social cycles }\end{array}$ & $\begin{array}{l}\text { Privacy in social } \\
\text { networks in close } \\
\text { distance }\end{array}$ \\
\hline $\begin{array}{l}\text { Q8: The behaviour of the application changes automatically, based } \\
\text { on your distance from another user }\end{array}$ & $\begin{array}{l}\text { Pervasiveness based } \\
\text { on social proximity }\end{array}$ & $\begin{array}{l}\text { Privacy in social } \\
\text { networks in close } \\
\text { distance }\end{array}$ \\
\hline $\begin{array}{l}\text { Q9: The behaviour of the application changes automatically based } \\
\text { on the place or area you are }\end{array}$ & $\begin{array}{l}\text { Pervasiveness based } \\
\text { on location proximity }\end{array}$ & $\begin{array}{l}\text { Privacy in social } \\
\text { networks in close } \\
\text { distance }\end{array}$ \\
\hline $\begin{array}{l}\text { Q10: Allow/deny certain persons or groups to have access } \\
\text { automatically in specific activities, when in proximity }\end{array}$ & $\begin{array}{l}\text { Pervasiveness based } \\
\text { on Activity Theory }\end{array}$ & $\begin{array}{l}\text { Privacy in social } \\
\text { networks in close } \\
\text { distance }\end{array}$ \\
\hline
\end{tabular}

*(you may disable this functionality whenever you want)

The criteria selected to evaluate each question covered a range of aspects, including clearness, innovativeness, virality, easiness, tendency to use, pleasure and urgency; Certain TAM criteria were altered to include factors of (a) innovativeness of the solution as perceived by the users, (b) clarity of the concept, and (c) virality of the concept as it is vital for a social network to grow mouth to mouth. Each criterion was rated based on a scale from 0 to 3 , without a middle score to force the audience to express a clear preference (see Table 3).

Table 3: The criteria and the relative responses available, under which every question was evaluated

\begin{tabular}{|l|l|l|l|l|l|}
\hline Evaluation Criteria (TAM3) & $\begin{array}{l}\text { Relative responses available } \\
\text { to users }\end{array}$ & $\begin{array}{l}\text { Not at } \\
\text { all }\end{array}$ & $\begin{array}{l}\text { Possibly } \\
\text { No }\end{array}$ & $\begin{array}{l}\text { Possibly } \\
\text { Yes }\end{array}$ & Definitely \\
\hline C0: Job & R0: Area of employment & - & - & - & - \\
\hline C2: Clarity* & $\begin{array}{l}\text { R1: It is clear to me what it } \\
\text { does }\end{array}$ & 0 & 1 & 2 & 3 \\
\hline C1: Innovation/Uniqueness* & R2: Sounds innovative/new & 0 & 1 & 2 & 3 \\
\hline C5: Perceived Usefulness & R3: I need it & 0 & 1 & 2 & 3 \\
\hline C6: Ease of use & R4: Sounds easy & 0 & 1 & 2 & 3 \\
\hline $\begin{array}{l}\text { C7: Behavioural Intention to } \\
\text { Use }\end{array}$ & R5: I would use it & 0 & 1 & 2 & 3 \\
\hline C4: Perceived Enjoyment & R6: Sounds pleasant & 0 & 1 & 2 & 3 \\
\hline $\begin{array}{l}\text { C7: Behavioural Intention to } \\
\text { Use }\end{array}$ & R7: I want it now & 0 & 1 & 2 & 3 \\
\hline C8: Virality & $\begin{array}{l}\text { R8: I would suggest it to } \\
\text { friends }\end{array}$ & 0 & 1 & 2 & 3 \\
\hline C3: Anxiety & $\begin{array}{l}\text { Asked gpecific features F1- } \\
\text { F9 (see Table 4) }\end{array}$ & 0 & 1 & 2 & 3 \\
\hline C9: Actual Usage & & 1 & 2 & 3 \\
\hline
\end{tabular}

(with * the criteria merged with the relative family of criteria in TAM3 are marked)

To evaluate Actual Usage (C9) of a Social Agent, a set of functionalities were generated to make it clearer what a Social Agent may do in action, as it was decided not to offer a case-specific prototype at this level of evaluation. A set of nine different features was generated, having both social and pervasive characteristics (see Table 4), covering the social networking needs of users (based on work of Xu C. et al. [73]) for (a) immediate access to people with existing social connections (e.g. friends and family), (b) coordination and organization of group of people (e.g. events), and (c) affection to other persons as the main three reasons that someone uses social networking, while (d) leisure time is a 
minor reason for using social networks. Last but not least, (e) the need for social presence and communication is worth-mentioning as well. For each feature, interviewees were asked whether they like it or not in a social networking application, based in the same scale from 0 to 3 (i.e. from not at all to definitely).

Table 4: The nine features asked to evaluate actual usage (C9)

\begin{tabular}{|l|l|}
\hline Specific features to evaluate actual usage (C9) & Social needs [73] \\
\hline $\begin{array}{l}\text { F1 - Transmission: Share automatically with your network an activity that a friend has just } \\
\text { completed, to allow your friend to reach a broader audience (i.e. push virality) }\end{array}$ & Leisure \\
\hline $\begin{array}{l}\text { F2 - Broadcast: Automatically announce to specific group of people the outcome of } \\
\text { predefined activities }\end{array}$ & Leisure \\
\hline $\begin{array}{l}\text { F3 - Share: A human activity may be shared to existing social media platforms automatically, } \\
\text { under certain user rules (e.g. certain context or types of activities), to reach user's existing } \\
\text { network of friends anytime, wherever they are }\end{array}$ & $\begin{array}{l}\text { Immediate } \\
\text { access }\end{array}$ \\
\hline $\begin{array}{l}\text { F4 - Schedule: Schedule activity in the future, and invite automatically friends in proximity } \\
\text { with similar activities to increase acceptance rate }\end{array}$ & Coordination \\
\hline $\begin{array}{l}\text { F5 - Similar interests: Exchange with people in proximity similar activities to engage } \\
\text { discussion }\end{array}$ & Affection \\
\hline $\begin{array}{l}\text { F6 - Help: Ask help on specific types of activities (e.g. learn coding), and get notified when } \\
\text { someone in proximity has experience }\end{array}$ & Affection \\
\hline F7 - Compare: Compare with people in proximity similar activities & Affection \\
\hline F8 - Statistics: Monitor analytics on the activities the user has undertaken (privately) & Leisure \\
\hline F9 - New Activities: Announce to friends in proximity any new activity you have participated & Social Presence \\
\hline
\end{tabular}

Figure 15 gives an overview of how the criteria and the functionalities are expected to be correlated, according to the TAM3 theory. It is clearer in this diagram how the correlation between Behavioural Intention to Use (C7) and Actual Usage (C9) was investigated, in a matter of different functionalities and not as a concrete application.

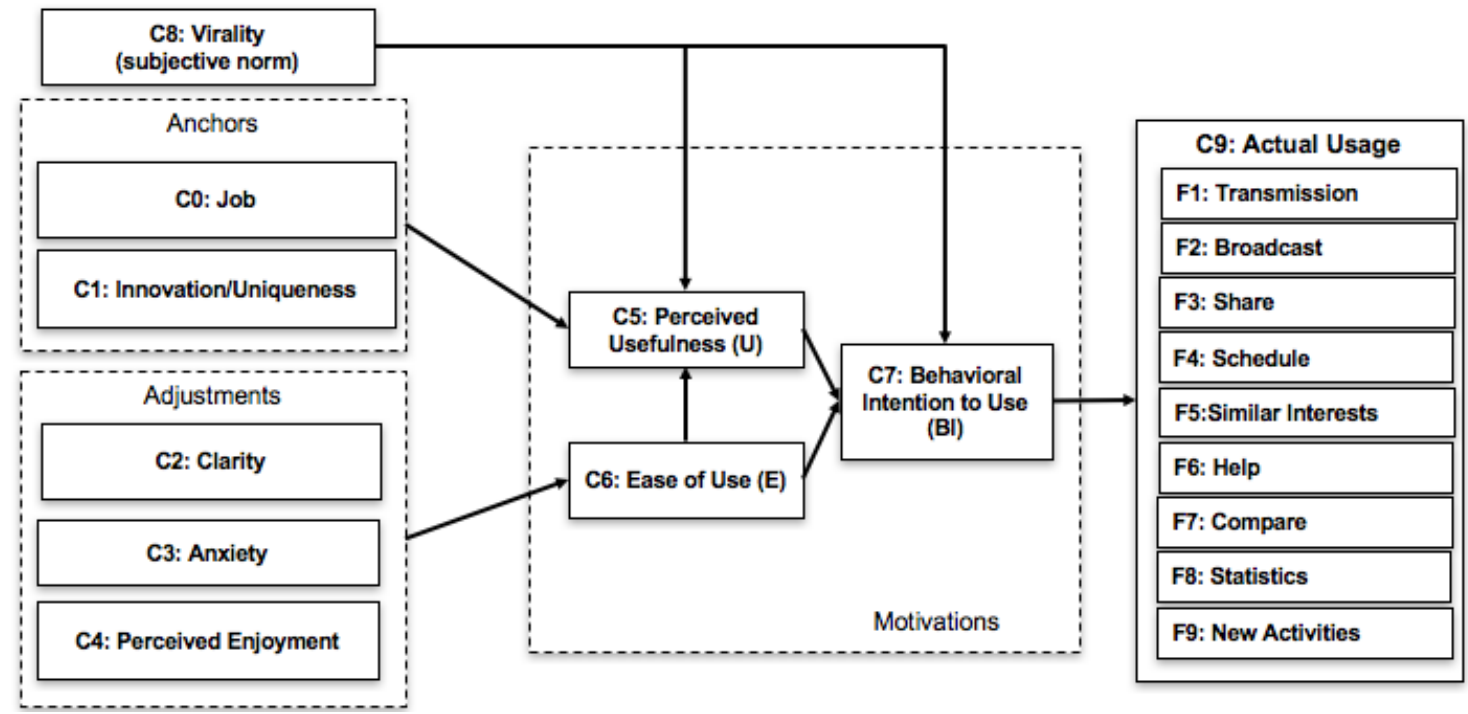

Figure 15: Criteria and functionalities expected to correlate based on the methodology

\subsubsection{High-Level Evaluation Implementation and Analysis}

The evaluation process was based on an online survey distributed among 200 people in Greece. For the selected country, $50 \%$ of the population had Internet access by Q1 of 2015 . From this group of population, $65.7 \%$ of them $(3,516,735$ people) have access to websites of social networking, mainly young people [27]. Additionally, Greece is a conservative audience for social media platforms, compared to the US and UK market, so the concept of the Social Agent was tested against a rather challenging audience. The questionnaire (an anonymised English version of which can be found in (Site 26)) was answered voluntarily, and targeted channels used by both people who use social networks and people who do not. Answers were accepted online following the gender as the grouping dimension to monitor the sampling groups. Overall 200 different answers were collected, out of which 197 were fully answered. Based on the size of population of 5,352,717 Greeks with Internet access, and confidence 95\%, the statistical error is estimated on $\pm 6.93 \%$. After the collection of the responses, the analysis included two phases, (a) a quantitative analysis of the statistical results directly, and (b) a secondary analysis to identify any correlation among the different criteria and functions as described in the methodology in section 4.1.1. After the analysis, the outcomes are the following (see Table 5): 
- $\quad$ The concept of the Social Agent does not make the sample anxious (R9), something unexpected as the social pervasive connections were considered intrusive for common social media users. Nevertheless, the sample answered that they do not need it (R3), or want it immediately (R7); this is reasonable for the area of social networking, where the need of communication is already covered from typical social media platforms and users typically look for fun and pleasure in niche platforms.

- The personal analytics (Q4) and the pervasive services (Q5) seem to be the only functions that the majority would use (R5). Exposing user to people and devices in proximity (Q6) was the only feature that the majority clearly rejected, while for the rest functions (Q1-Q4) there is a positive but not clear stance towards actual usage.

- The only question that made the sample feel uncomfortable (R6) was the collection of data from the web (Q2), in comparison to the collection of data from daily activities (Q3) which was judged as pleasant from the majority. That could mean that people consider their online presence more sensitive or that they are not aware of devices tracking daily activities.

- $\quad$ All other features were clear to the audience (R1), sounded innovative or new (R2), and would be suggested to friends in most cases (R8), apart from the discovery in proximity (Q6).

- Overall, the most important concept, the one for close-distance communication based on activities (Q1) would be used (R5) by $51.3 \%$ of the sample, while $58.4 \%$ are positive to suggest it to a friend (R8). All the supporting features except for notification for users in close distance (Q2-Q5) were not dropped for usage (R5) and they could possibly increase the acceptance rate of the social agent if combined.

Table 5: The evaluation of social networking in close distance under each criterion, for responders (Q1-Q6)

\begin{tabular}{|c|c|c|c|c|c|c|c|c|c|c|}
\hline & Value & R1 & $\mathrm{R} 2$ & R3 & R4 & R5 & R6 & R7 & $\mathrm{R} 8$ & R9 \\
\hline \multirow[t]{2}{*}{ Q1 } & {$[0-1]$} & $8.1 \%$ & $29.9 \%$ & $87.8 \%{ }^{*}$ & $18.8 \%$ & $48.7 \%$ & $34.5 \%$ & $88.8 \%{ }^{*}$ & $41.6 \%$ & $74.6 \%{ }^{*}$ \\
\hline & [2-3] & $91.9 \% *$ & $70.1 \%$ * & $12.2 \%$ & $81.2 \%{ }^{*}$ & $51.3 \%$ ** & $65.5 \%$ * & $11.2 \%$ & $58.4 \%{ }^{*}$ & $25.4 \%$ \\
\hline \multirow[t]{2}{*}{ Q2 } & {$[0-1]$} & $8.6 \%$ & $42.6 \%$ & $81.2 \%{ }^{*}$ & $20.8 \%$ & $47.7 \%$ & $61.9 \%{ }^{*}$ & $86.8 \%{ }^{*}$ & $53.3 \%$ ** & $71.6 \%$ * \\
\hline & [2-3] & $91.4 \%{ }^{*}$ & $57.4 \%$ * & $18.8 \%$ & $79.2 \%$ * & $52.3 \%$ ** & $38.1 \%$ & $13.2 \%$ & $46.7 \%$ & $28.4 \%$ \\
\hline \multirow[t]{2}{*}{ Q3 } & {$[0-1]$} & $10.7 \%$ & $41.1 \%$ & $63.5 \%$ * & $27.9 \%$ & $45.2 \%$ & $40.6 \%$ & $79.2 \%$ * & $40.1 \%$ & $73.6 \%$ * \\
\hline & [2-3] & $89.3 \%$ * & $58.9 \%$ * & $36.5 \%$ & $72.1 \%$ * & $54.8 \%$ * & $59.4 \%$ * & $20.8 \%$ & $59.9 \%$ * & $26.4 \%$ \\
\hline \multirow[t]{2}{*}{ Q4 } & {$[0-1]$} & $8.1 \%$ & $39.6 \%$ & $60.4 \% *$ & $25.4 \%$ & $39.6 \%$ & $30.5 \%$ & $72.6 \%{ }^{*}$ & $35.5 \%$ & $85.8 \%{ }^{*}$ \\
\hline & [2-3] & $91.9 \%{ }^{*}$ & $60.4 \%$ * & $39.6 \%$ & $74.6 \%$ * & $60.4 \% *$ & $69.5 \%$ * & $27.4 \%$ & $64.5 \%{ }^{*}$ & $14.2 \%$ \\
\hline \multirow[t]{2}{*}{ Q5 } & {$[0-1]$} & $9.1 \%$ & $20.3 \%$ & $50.3 \%$ ** & $31.0 \%$ & $27.9 \%$ & $24.4 \%$ & $65.0 \%$ * & $27.9 \%$ & $86.8 \%$ * \\
\hline & [2-3] & $90.9 \%{ }^{*}$ & $79.7 \%$ * & $49.7 \%$ & $69.0 \%{ }^{*}$ & $72.1 \%^{*}$ & $75.6 \%$ * & $35.0 \%$ & $72.1 \%^{*}$ & $13.2 \%$ \\
\hline \multirow[t]{2}{*}{ Q6 } & {$[0-1]$} & $9.1 \%$ & $17.8 \%$ & $89.8 \%{ }^{*}$ & $20.3 \%$ & $74.6 \%{ }^{*}$ & $48.2 \%$ & $92.9 \%{ }^{*}$ & $60.9 \%^{*}$ & $73.6 \%{ }^{*}$ \\
\hline & [2-3] & $90.9 \% *$ & $82.2 \%{ }^{*}$ & $10.2 \%$ & $79.7 \%{ }^{*}$ & $25.4 \%$ & $51.8 \%$ ** & $7.1 \%$ & $39.1 \%$ & $26.4 \%$ \\
\hline
\end{tabular}

${ }^{\star}:$ Clear preference on total positive or negative, ${ }^{\star *}$ : Not clear preference

The outcomes for the privacy settings and the pervasive character of the social agent are the following (see Table 6):

- $\quad$ For the basic settings of social agent (Q7), the sample does not consider it as a need (R3, R7), something reasonable as they do not evaluate positively the basic concept as well (Q1), but on every other criterion they are positive. This means that the Social Agent should develop sophisticated privacy settings.

- $\quad$ All the other pervasive settings and privacy features (Q8-Q10) are judged as clear (R1), innovative (R2) and easy to use (R4), but do not show acceptance on all the other criteria. This may imply that the audience is unfamiliar with the idea of pervasiveness.

Table 6: The evaluation of privacy in social networks in close distance, under each criterion

\begin{tabular}{|c|c|c|c|c|c|c|c|c|c|c|}
\hline & Value & R1 & $\mathrm{R} 2$ & R3 & R4 & R5 & $\mathrm{R} 6$ & R7 & R8 & R9 \\
\hline \multirow[t]{2}{*}{ Q7 } & {$[0-1]$} & $8.1 \%$ & $41.6 \%$ & $68.0 \%{ }^{*}$ & $20.8 \%$ & $37.6 \%$ & $37.1 \%$ & $79.2 \%{ }^{*}$ & $38.1 \%$ & $79.7 \%{ }^{*}$ \\
\hline & [2-3] & $91.9 \%$ * & $58.4 \%$ * & $32.0 \%$ & $79.2 \%$ * & $62.4 \%{ }^{*}$ & $62.9 \% *$ & $20.8 \%$ & $61.9 \%{ }^{*}$ & $20.3 \%$ \\
\hline \multirow[t]{2}{*}{ Q8 } & {$[0-1]$} & $34.5 \%$ & $15.2 \%$ & $90.4 \%{ }^{*}$ & $42.1 \%$ & $72.1 \%{ }^{*}$ & $51.3 \% \%^{* *}$ & $93.9 \%{ }^{*}$ & $61.4 \%{ }^{*}$ & $72.1 \%{ }^{*}$ \\
\hline & [2-3] & $65.5 \%$ * & $84.8 \%$ * & $9.6 \%$ & $57.9 \%$ * & $27.9 \%$ & $48.7 \%$ & $6.1 \%$ & $38.6 \%$ & $27.9 \%$ \\
\hline \multirow[t]{2}{*}{ Q9 } & {$[0-1]$} & $19.3 \%$ & $22.8 \%$ & $83.2 \%{ }^{*}$ & $33.0 \%$ & $62.4 \%^{*}$ & $44.2 \%$ & $91.4 \%{ }^{*}$ & $51.3 \%$ ** & $82.2 \%{ }^{*}$ \\
\hline & [2-3] & $80.7 \%$ * & $77.2 \%{ }^{*}$ & $16.8 \%$ & $67.0 \%$ * & $37.6 \%$ & $55.8 \%$ ** & $8.6 \%$ & $48.7 \%$ & $17.8 \%$ \\
\hline \multirow[t]{2}{*}{ Q10 } & {$[0-1]$} & $10.7 \%$ & $45.7 \%$ & $71.6 \%$ * & $20.3 \%$ & $51.3 \%$ ** & $49.2 \%$ & $81.2 \%$ * & $45.7 \%$ & $81.7 \%$ * \\
\hline & [2-3] & $89.3 \%$ * & $54.3 \%$ ** & $28.4 \%$ & $79.7 \%$ * & $48.7 \%$ & $50.8 \%$ ** & $18.8 \%$ & $54.3 \%$ ** & $18.3 \%$ \\
\hline
\end{tabular}

*: Clear preference on total positive or negative, ${ }^{* *}$ : Not clear preference 
The statistical analysis of the features (see Table 7) revealed a clear positive view for the features of asking for help with $72.1 \%$ (F6), looking for the statistics with 70.6\% (F8), and transmitting activities to friends with $65.5 \%(\mathrm{~F} 1)$. With regard to broadcasting activities on friends (F2), scheduling activities and inviting people in proximity (F4) and finding common interests with people in proximity (F5), there is no clear majority, with $54.8 \%, 44.2 \%$ and $43.7 \%$ positive views respectively. Those results are quite high and promising for the area of pervasive social networking.

Table 7: The evaluation of features from interviewees that responded to measure actual usage (C9)

\begin{tabular}{|l|l|l|l|l|l|l|}
\hline & 0 & 1 & 2 & 3 & Total Negative & Total Positive \\
\hline F1: Transmission & $11.7 \%$ & $22.8 \%$ & $49.7 \%$ & $15.7 \%$ & $34.5 \%$ & $65.5 \%^{*}$ \\
\hline F2: Broadcast & $17.3 \%$ & $27.9 \%$ & $35.5 \%$ & $19.3 \%$ & $45.2 \%$ & $54.8 \%^{* *}$ \\
\hline F3: Share & $41.1 \%$ & $42.1 \%$ & $12.7 \%$ & $4.1 \%$ & $83.2 \%{ }^{*}$ & $16.8 \%$ \\
\hline F4: Schedule & $21.3 \%$ & $34.5 \%$ & $31.5 \%$ & $12.7 \%$ & $55.8 \% \%^{* *}$ & $44.2 \%$ \\
\hline F5: Similar interests & $23.4 \%$ & $33.0 \%$ & $34.5 \%$ & $9.1 \%$ & $56.3 \% \%^{* *}$ & $43.7 \%$ \\
\hline F6: Help & $8.1 \%$ & $19.8 \%$ & $53.3 \%$ & $18.8 \%$ & $27.9 \%$ & $72.1 \%^{*}$ \\
\hline F7: Compare & $25.4 \%$ & $37.6 \%$ & $28.9 \%$ & $8.1 \%$ & $62.9 \%^{*}$ & $37.1 \%$ \\
\hline F8: Statistics & $9.1 \%$ & $20.3 \%$ & $46.2 \%$ & $24.4 \%$ & $29.4 \%$ & $70.6 \%^{*}$ \\
\hline F9: New Activities & $31.5 \%$ & $39.1 \%$ & $23.4 \%$ & $6.1 \%$ & $70.6 \%^{*}$ & $29.4 \%$ \\
\hline
\end{tabular}

${ }^{*}:$ Clear preference on total positive or negative (if summarised), ${ }^{* *}$ : Not clear preference

The secondary analysis examined any correlation among the selected criteria. Initially, responses (R1-R9) were merged under criteria ( $\mathrm{C} 1-\mathrm{C} 8)$. Criterion $\mathrm{C} 0$ was grouped based on the industry of the job, while for criteria form $\mathrm{C} 1$ to C8 the mean value of the responses (0-3) for all the questions was used (Q1-Q10) to generate a value to correlate, including the questions on privacy settings (Q7-Q10). Table 8 shows the results of the correlation analysis among the selected criteria under the methodology. A slight difference between the correlations among the criteria expected to correlate based on TAM and the identified correlations (i.e. score over 0.4 ) may be noticed.

Table 8: The results from the correlation analysis among the selected criteria

\begin{tabular}{|l|l|l|l|l|l|l|l|l|l|}
\hline${ }^{*}: \mathrm{p}<=0.01$ & C0 & C1 & C2 & C3 & C4 & C5 & C6 & C7 & C8 \\
\hline C0: Job & - & 0.12 & 0.17 & -0.09 & 0.06 & $0.05(x c)$ & 0.01 & 0.09 & 0.07 \\
\hline $\begin{array}{l}\text { C1: } \\
\text { Innovation/Uniqueness }\end{array}$ & - & - & 0.33 & -0.26 & $0.5^{*}$ & $0.48^{*}(\mathrm{xc})$ & 0.33 & $0.5^{*}$ & $0.51^{*}$ \\
\hline C2: Clarity & - & - & - & -0.35 & 0.3 & 0.17 & $0.48^{*}(\mathrm{xc})$ & 0.22 & 0.32 \\
\hline C3: Anxiety & - & - & - & - & -0.44 & -0.19 & $-0.35(\mathrm{xc})$ & -0.27 & -0.37 \\
\hline C4: Perceived Enjoyment & - & - & - & - & - & 0.7 & $0.55^{\star}(\mathrm{xc})$ & $0.8^{\star *}$ & $0.83^{\star *}$ \\
\hline C5: Perceived Usefulness & - & - & - & - & - & - & $0.37(\mathrm{xc})$ & $0.91^{* *}(\mathrm{xc})$ & $0.7^{* *}(\mathrm{xc})$ \\
\hline C6: Ease of use & - & - & - & - & - & - & - & $0.4^{*}(\mathrm{xc})$ & $0.46^{*}$ \\
\hline $\begin{array}{l}\text { C7: Behavioural Intension } \\
\text { to Use }\end{array}$ & - & - & - & - & - & - & - & - & $0.8^{* *}(\mathrm{xc})$ \\
\hline C8: Virality & - & - & - & - & - & - & - & - & - \\
\hline
\end{tabular}

${ }^{*}$ : Correlation, ${ }^{* *}$ : Strong correlation, $(\mathrm{xc})$ : expected correlation under TAM

Up next, the correlation between the Behavioural Intention $(\mathrm{BI})$ to Use of the interviewees $(\mathrm{C} 7)$ and the Actual Usage (C9) was examined, separately for each social functionality (F1-F9), as seen in Table 9. The reason for examining each feature separately for correlation is that the selected features are indicative, and on implementation time they may not be combined in one solution. Correlation was found for F2, F4, F5, F6, F7 and F9. However, it is important to compare the correlation found with the statistical preference of the interviewees as seen in Table 9; Asking for help on specific activities (F6) was judged as positive, while it was correlated with intention to use of the concept of the Social Agent. No other clearly preferable feature is correlated with $\mathrm{Bl}$ to use. From the rest of the features, F2, F4, F5 were found preferable in the area of statistical error, while they were correlated with Intention to Use.

Table 9: Correlating intention to use, with proposed interactions

\begin{tabular}{|l|l|l|}
\hline Interactions for Actual Usage (C9) & C7: Behavioural Intention (BI) to Use & Relevant positive statistics \\
\hline F1: Transmission & 0.29 & $65.5 \%$ \\
\hline F2: Broadcast & $0.44^{*}$ & $54.8 \%$ \\
\hline F3: Share & 0.32 & $16.8 \%$ \\
\hline F4: Schedule & $0.47^{*}$ & $44.2 \%$ \\
\hline F5: Similar interests & $0.57^{*}$ & $43.7 \%$ \\
\hline F6: Help & $0.4^{*}$ & $72.1 \%$ \\
\hline F7: Compare & $0.41^{*}$ & $37.1 \%$ \\
\hline F8: Statistics & 0.34 & $70.6 \%$ \\
\hline F9: New Activities & $0.44^{*}$ & $29.4 \%$ \\
\hline
\end{tabular}

*: Correlation

losif Alvertis

Evmorfia Biliri Fenareti Lampathaki Dimitris Askounis 
Figure 16 shows the actual TAM diagram after the evaluation of the Social Agent, showing into one diagram the correlations that were found among the different criteria and the proposed interactions. Overall, the only strong correlation is among the Usefulness and the Tendency to Use with 0.91. People that consider the concept of pervasive social networking based on activities as Innovative, see usefulness on it and have Tendency to Use it. People that consider the concept as Pleasant tend to Use it and Suggest it to friends.
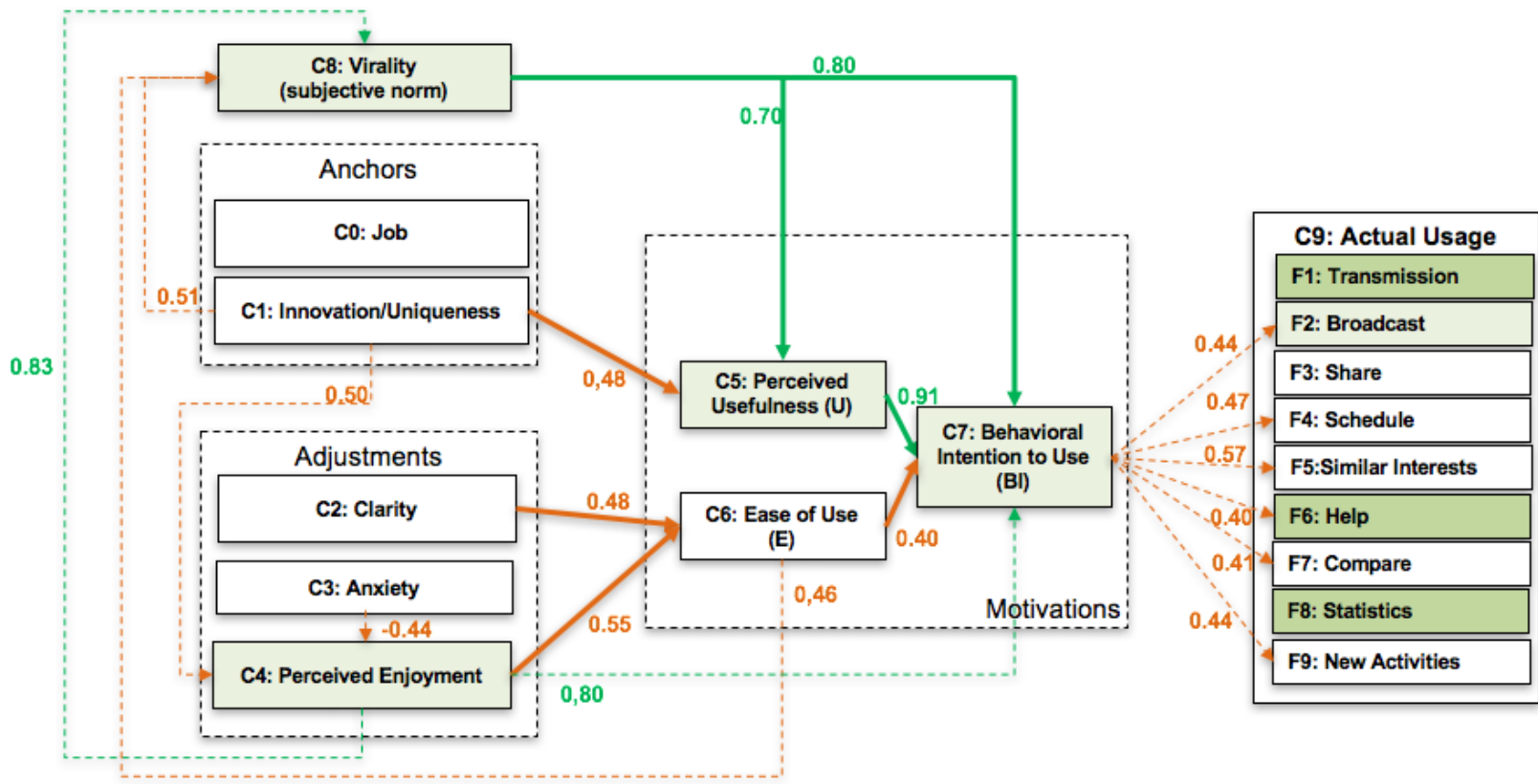

Figure 16: The final TAM model (green lines for strong correlations, dashed lines for not expected correlations)

Overall, if a Social Agent were to be released in the market, the suggested interactions/functions should be: (F6) asking for help, (F5) common interests in proximity, (F4) scheduling activities in proximity, (F2) broadcasting activities to specific groups. Those functionalities have both high proportions and correlation. For the rest of the popular features, they should not be characterised as indifferent for interviewees, but perceptually users may not be attracted by the broader concept of the Social Agent. Initially, focus should be given on features that correlate with intention to use. Then, strategically, if someone wants to attract people who are not fond of the concept of pervasive social networking (i.e. mainstream segments) and reach faster community growth, transmission of activities (F1) and seeing activity analytics (F8) may be the best options to begin with; selecting popular features may attract mainstream users for reasons different from pervasive agency in social interactions.

\subsection{Prototyping a Social Agent}

To evaluate the technical feasibility of a Social Agent, a prototype was designed and developed (Site 4). The main decisions made for the prototype were to (a) use a centralised architecture to decrease the response time of the application, and (b) use modern technologies (i.e. Django framework, HTML5 with responsive UI, Postgres, Heroku server) to increase the quality of the prototype. At the end, the concept of the Social Agent should be independent from the technical implementation, provided that it meets the main principles of pervasiveness, that it completes the goal of social networking, as well as the data and service interoperability as described in Section 3.

Figure 17 presents the architecture of the developed prototype, organised in the following layers:

- Data input: Users could define their topics of interest (e.g. pop music, soccer etc.), as well as to invite friends in the platform and declare a social connection with them. This option improved the accuracy of user matching on topics not collected from external resources, and solved the cold-start problem (i.e. user interests and social connections in figure 17. Users could also connect their profile with existing cloud-based services to reuse their data while establishing new social connection with their Social Agents; connectors with Facebook, Runkeeper, Twitter, YouTube and FitBit were developed, to highlight the symbiotic character of the Agent with existing social media platforms. Additionally, users could define what was their undertaken activity (see figure 18), to enable better interactions with services and people in proximity (i.e. user input on current activity in figure 17. The users were not allowed to enter historic data manually into the application, not to be able to alter the behaviour of the Social Agent. On the implemented input view, there was a dictionary of available activities to choose from, and a list of suggested activities generated by the system based on the social context (i.e. in figure 18 with blue label) of the application and based on the personal context (i.e. in figure 18 with brown label) of the user. This feature was indicative of the predictive character of the output of a Social Agent, and its strong pervasive character. Finally, a printed form was given to test users of the application, together with a dictionary of activities, and they were asked to create two daily logs of their lives, for the days 
of their choice. This input was used to identify users' routines, to identify activities that users cannot synchronise from external services (e.g. driving, working or discussing) and describe the hierarchy on specific activities implied by users. Then, this log was inserted into the user profile by the administrator of the application.

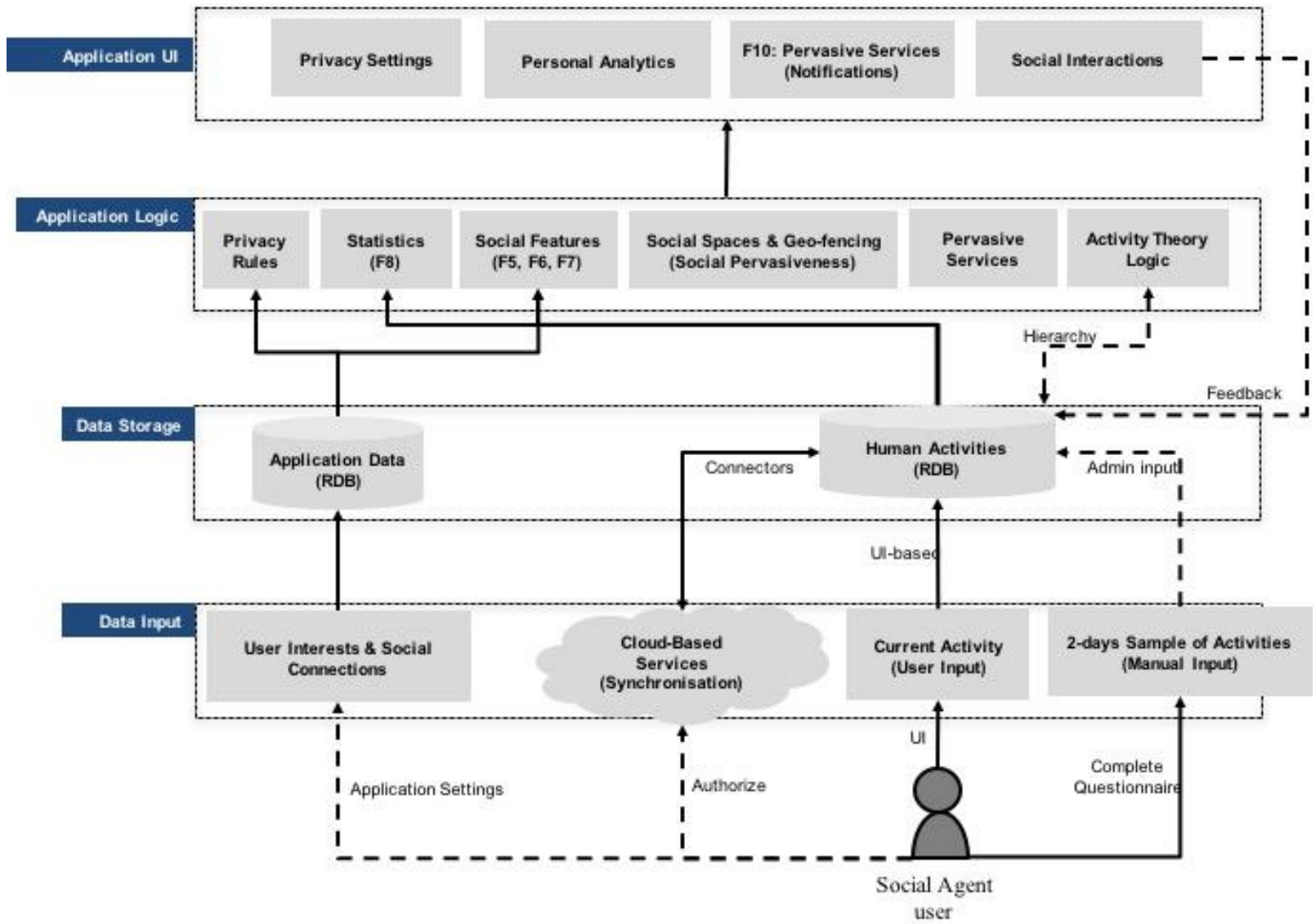

Figure 17: The architecture of the social agent prototype

- Data storage: All activity-related data were stored under the schema described in section 3.3 (i.e. human activities RDB in figure 17, while users' preferences and interests were stored in a case-specific schema (i.e. application data RDB in figure 17, properly connected with the activity schema under the profile of the actor of the activity.

- Application logic: This layer implemented an instance of the Social Agent with specific social features. Certain decisions were made in order to improve user experience, based on the theoretical analysis that had been made in Section 3. Thus, initially the social spaces were designed based on distance between users, as described in Section 3.2, and certain pervasive social interactions were mapped to each space (see figure 19); Each space was identified based on the networking protocol used: (a) the intimate space where two users were able to match their common activities was enabled by NFC (F5), (b) the personal space was enabled by Bluetooth and allowed two users to compare their activities (F7), (c) the social space was enabled under the same Wi-Fi network and allowed a user to ask help on a certain topic, if a user with relative experience was in proximity (F6), and (d) the public space for people further than 7.5 meters allowed users to send a notification in order to meet each other. Moreover, privacy settings allowed users to easily hide from strangers, thus not to use the pervasive service of the social agent to connect with people in proximity, or hide completely from the network of users even if they are friends. The Activity Theory logic allowed modelling different types of input in different hierarchy levels (I.e. routines in activity level, cloud-based entries in actions and digital traces in operations), while the pervasive services were triggered based on the undertaken activity of the user to contact with APIs of cloud-based services and bring relative content (e.g. power-songs from YouTube when running). 


\section{$\leftarrow \quad$ Set the activity you are upon now}

\begin{tabular}{l} 
\# Activity type \\
None (click to change it) \\
\hline running \\
exercising \\
working \\
drinking \\
dinner \\
breakfast \\
soccer \\
partying \\
driving
\end{tabular}
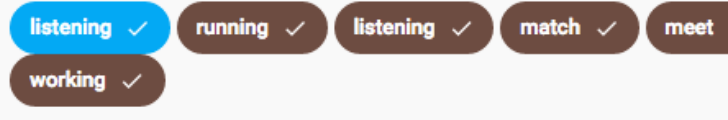

Figure 18: An activity entry happens by the time it happens, and contextual suggestions are also visible

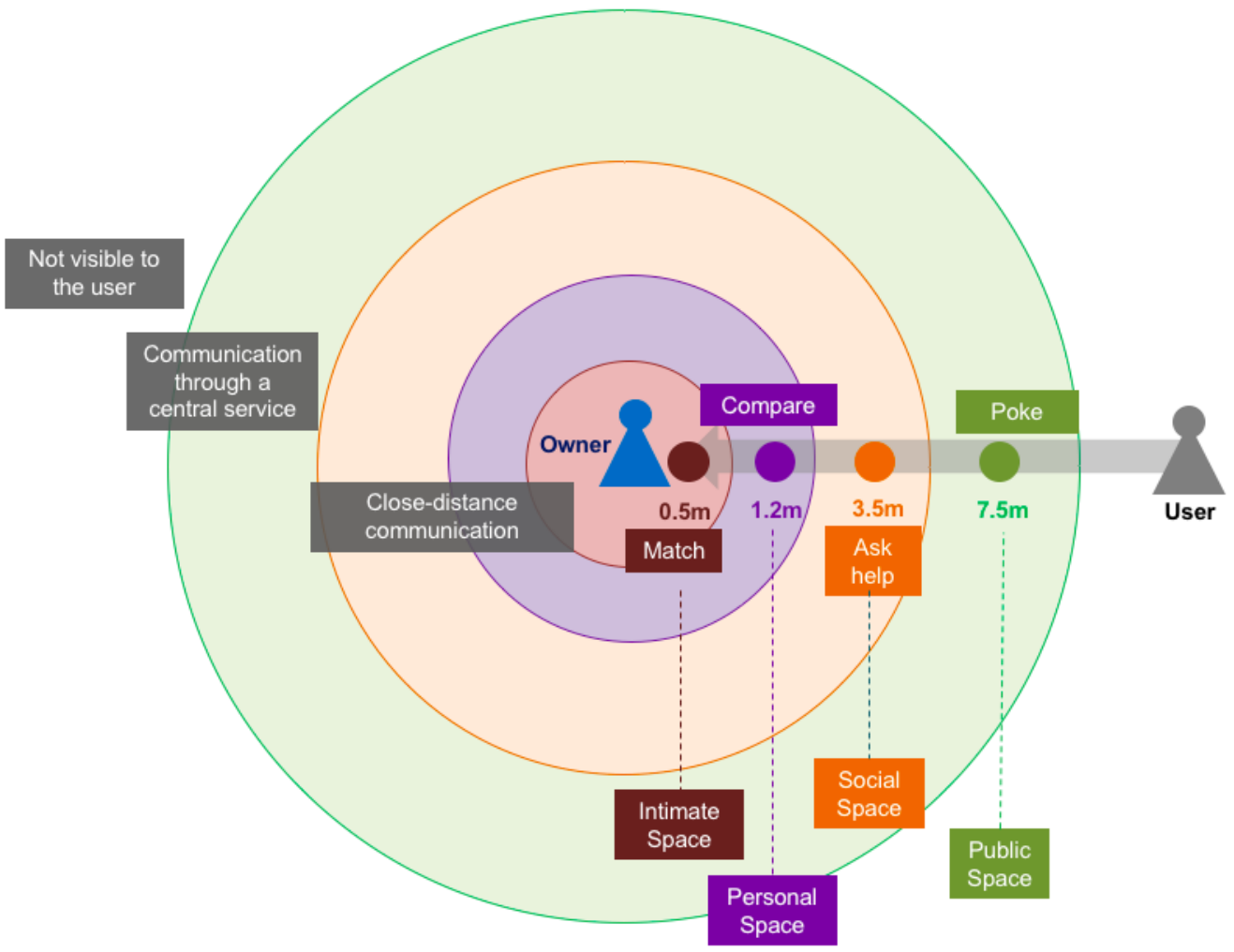

Figure 19: The physical distance enables pervasiveness on the personal social agent prototype

- $\quad$ Application UI: The social interactions were put in the landing screen of the application, with users in proximity grouped in different social spaces indicated with different labels and colours (see figure 20). Privacy settings were easily visible in the initial screen, not hidden in preferences, as users should be able to change their privacy status easily. Statistics also were accessible easily through the initial screen, to enhance transparency with the users on what data were collected. Notifications informed users for available interactions with devices or pervasive services in proximity (a new feature F10 which was not circulated in the questionnaire), based on the activity context of the user (i.e. see figure 21 ) the pop-up notification calling the user for action), while users were also able to log their current activity and the topic where they need help on, to trigger the relative social interaction (F6). To make exchanging data on activities appealing to users, the modelling suggested in Activity Theory was used to highlight the contextual properties as important parts of information (see figure 22). Finally, for each social interaction that users triggered through the social agent application, the user's activity log was updated with a new entry for that new activity.

losif Alvertis

Evmorfia Biliri

Fenareti Lampathaki

Dimitris Askounis 


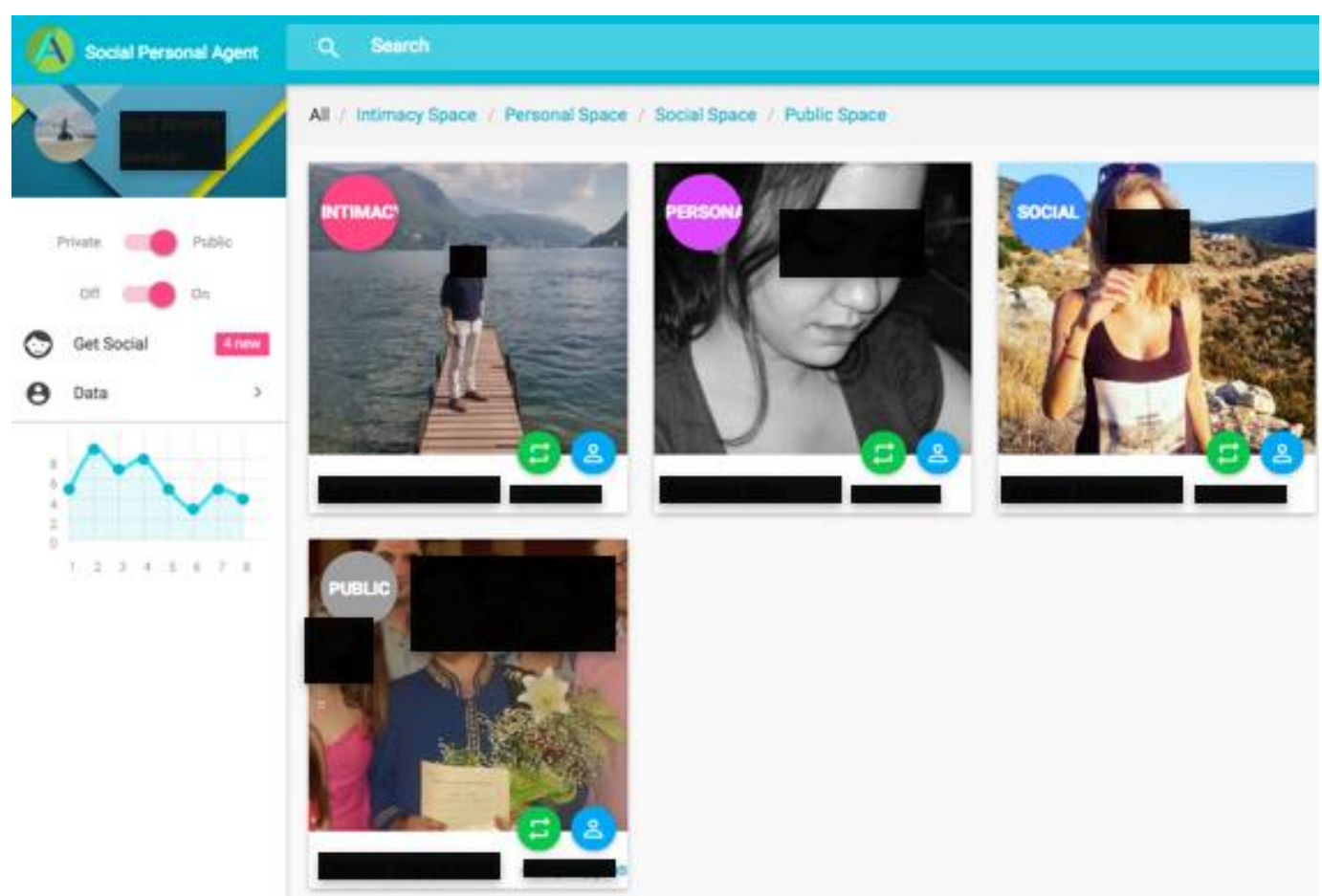

Figure 20: Users in proximity are presented to the owner in the central screen organised in different social spaces

\title{
Your activity now: running?
}

\author{
(1, This is your Running \#2 \\ Congratulations! \\ Keep up the hard work \\ Play Music \\ Beam Me Up (Radio Edit) \\ Cazzette - Runtastic Music - Running
}

Figure 21: Pervasive events based on the current activity context of the user

Overall, the goal of the prototype was to evaluate the feasibility of the Social Agent on implementation time. However, it is useful to document the reactions of the users while using the application. In brief, the developed application was tested on 20 university students. Users were willing to connect their accounts in the supported cloud-based services, and they liked the personal analytics screen. From the provided social features, they liked the comparison of their activities with other users in proximity. Possible future improvements identified are: (a) richer activity entries, as few services offer APIs to integrate users' activities and not every user used the selected services (e.g. RunKeeper), (b) development of mobile versions of the application with integration of notifications, as pervasiveness that may boost user engagement is weak on web applications, and (c) an integration of an activity resolver based on data from sensors to reduce required users' input on daily activities and routines. Finally, data collected through cloud-based services during the evaluation period were imported in a semantic repository under the SocAgOn ontology (Site 13), in order to evaluate it against its reasoning capabilities, while SPARQL queries were developed to implement the relative social functionalities of the prototype and validate the ontology in practice.

Iosif Alvertis

Evmorfia Biliri

Fenareti Lampathaki

Dimitris Askounis 


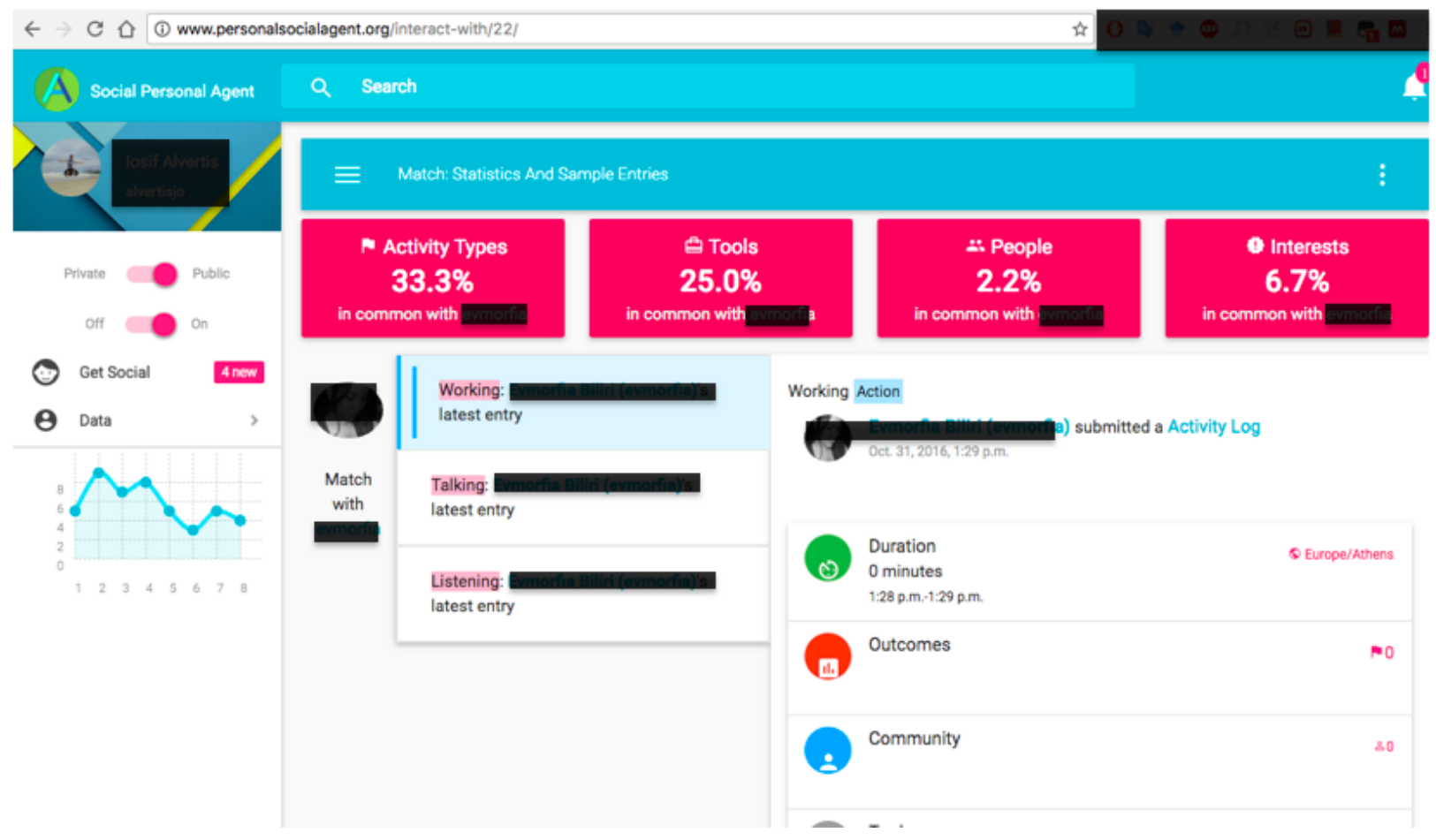

Figure 22: A match activities interaction between two users in personal social agent

\section{Discussion}

A Social Agent - as introduced in chapter 3 and validated in chapter 4 - is a symbiotic technology of social networking, collaborating with existing social media platforms and not competing with them. It tends to meet a need of augmented close-distance communication by standardising the way Pervasive Social Networking is performed. In this section, an extensive discussion takes place on how a Social Agent covers different aspects on the Value Chain of Pervasive Social Networking by positioning our work in the research area of social agents and social networks (section 5.1), then how it adds value in the research and business environment of social networking (section 5.2), and finally how it is positioned against relative research and solutions in the domain (section 5.3).

\subsection{Social Agent and Pervasive Social Networking}

On the research landscape of Pervasive Social Networking, as summarised in Figure 4, the Social Agent is a holistic approach that covers each phase of the data value chain, and it may be realised through various technologies (i.e. even existing ones as presented in the prototyping phase). As depicted in the case-specific value chain of Figure 23, the Social Agent may collect data from various resources, should map them under a common schema and define the dictionaries that make the entries interoperable across different implementations, may analyse the collected data (i.e. in its initial realisation only statistical analyses were presented in the prototype), and finally then should share the outcomes as raw statistics or generated user topics in order to create new social connections with people in proximity. In parallel, various other dimensions that define a social network have been described: the development of a new community of users was identified as an important factor for success (i.e. invitation of existing connections as social context and establishment of new ones), various tools to implement pervasive communication were discussed (i.e. wearable and mobile devices, agents and applications), different protocols to enable service exposure may be realised (i.e. NFC, Wi-Fi, Bluetooth, Internet etc.), different architectures may be implemented but a centralised one is preferable, the security layer should be always present, while privacy should be taken into consideration in various steps (e.g. allowing users to change easily privacy settings). 


\begin{tabular}{|c|c|c|c|c|c|}
\hline \multirow{2}{*}{$\frac{\text { Reuse connections }}{\text { Applications }}$} & \multicolumn{2}{|c|}{ Create new connections Community } & \multicolumn{2}{|c|}{ Mashup connections } & \multirow{7}{*}{\begin{tabular}{|l} 
Create \\
Value \\
from \\
Pervasive \\
Social \\
Networking
\end{tabular}} \\
\hline & Agents & Tools Mo & lobile devices & earable devices & \\
\hline Internet & Direct Exposure & Service Exposure & \multicolumn{2}{|c|}{$\begin{array}{c}\text { Close distance protocols } \\
\text { (NFC) }\end{array}$} & \\
\hline Centralized & Decentralized & Architecture & Distributed/P2 & Hybrid & \\
\hline Storage encryption & Authentication & Security & Authorization & Identity verification & \\
\hline \multicolumn{2}{|c|}{ On data types On connections On instances } & ances Privacy On & nn notifications On lo & cation On context & \\
\hline 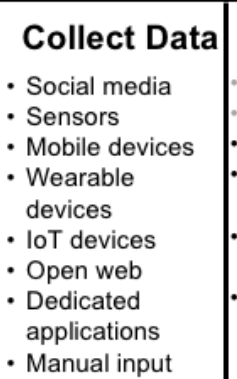 & $\begin{array}{l}\text { Map Data } \\
\text { - Transformation } \\
\text { - Uuration } \\
\text { - User Validation } \\
\text { Mix from different } \\
\text { resources } \\
\text { Map into } \\
\text { taxonomy } \\
\text { Map into schema }\end{array}$ & \begin{tabular}{rl|} 
& Analyze Data \\
- & Network analysis \\
- Signal Analysis \\
- Statistical \\
- Analysis \\
- Prediction \\
analysis \\
- Pattern \\
recognition
\end{tabular} & $\begin{array}{l}\text { Share Data } \\
\text { - Share instances } \\
\text { - Share statistics } \\
\text { - Share } \\
\text { interests/topics } \\
\text { - Share profiles }\end{array}$ & \begin{tabular}{|c|} 
Create \\
Connection \\
- Explicit/Manual \\
- Based on \\
context (e.g. \\
location \\
proximity) \\
- Based on \\
analysis (e.g. \\
social proximity)
\end{tabular} & \\
\hline
\end{tabular}

Figure 23: The data value chain for the proposed social agent

On the research landscape of social agency, as described by Carley K. and Newell A. [12], the introduced Social Agent may reach high levels of automatic response to status cues when trained with the proper data sets. and it includes a capability of reasoning to identify activity hierarchies and users' motivations. However, the developed prototype was focused on group making, by interacting with multiple agents and covering a bounded processing capability limited to the rules of Activity Theory. That gap between our hypothesis for the ideal Social Agent and the developed prototype is shown in figure 24, pointing out also different dimensions that future research may take, as well as the intermediate steps that a Social Agent may take before reaching the proposed form. Overall, the introduced social agent includes the characteristics that Kang D. et al [34] identified as critical for diffusion of a social networking service, as SNSs having a high degree of relationship extension represent a high-connection probability to users not already connected, and also, that SNSs having a high degree of shared interest have a relatively stronger external effect than other SNSs.

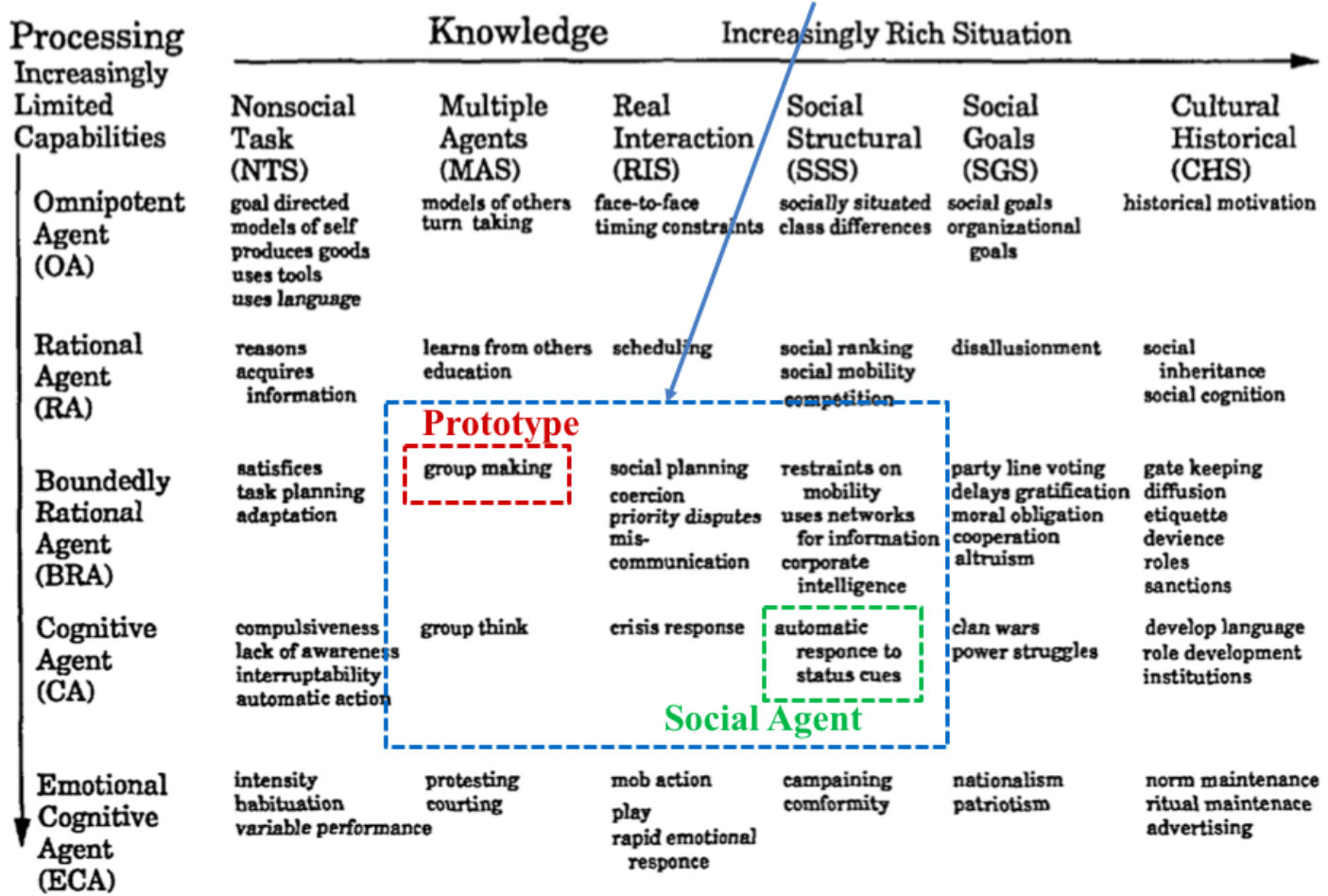

Figure 24: The Social Agent in the landscape of social agency (based on work by Carley, K., \& Newell, A) 
On social networks, contextual services have been recently considered as a core area of research and development for the industry, as seen in figure 2. The Social Agent implements a subcategory of a digital social network with strong pervasive characteristics and high level of agency, while its main goal remains to create social connections (figure 25). The suggested functionalities (F1-F9) used during the evaluation are only a subset of the possible functionalities that a Social Agent may implement, and some of them may not even show pervasive characteristics or features of agency; the reason is that in order to generate those features, our work was based on existing functionalities provided by current social media platforms, and a relevant matching with possible social features based on human activities. Nevertheless, during the feasibility test, functionalities at the core of the research in the area of pervasive social computing were selected, the popular feature of statistics (F8) was also selected to make it transparent for the users what data were collected, while a new feature for interacting with pervasive services was also added (i.e. F10 interaction with pervasive services based on human activities).

Apart from the work done in Pervasive Social Networking, with analysis (a) on modelling pervasive computing, (b) on social agency and (c) on technical progress in digital social networking, which took place in chapter 3 , the main contribution of our research was to combine those three domains in a novel way as never before in literature, under the Activity Theory, by using a three-layer methodology to promote data interoperability in social networking; a data interoperability that may lead not only to messaging interoperability, but also to architectural interoperability across different social media platforms and pervasive solutions (e.g. wearable devices and loT networks).

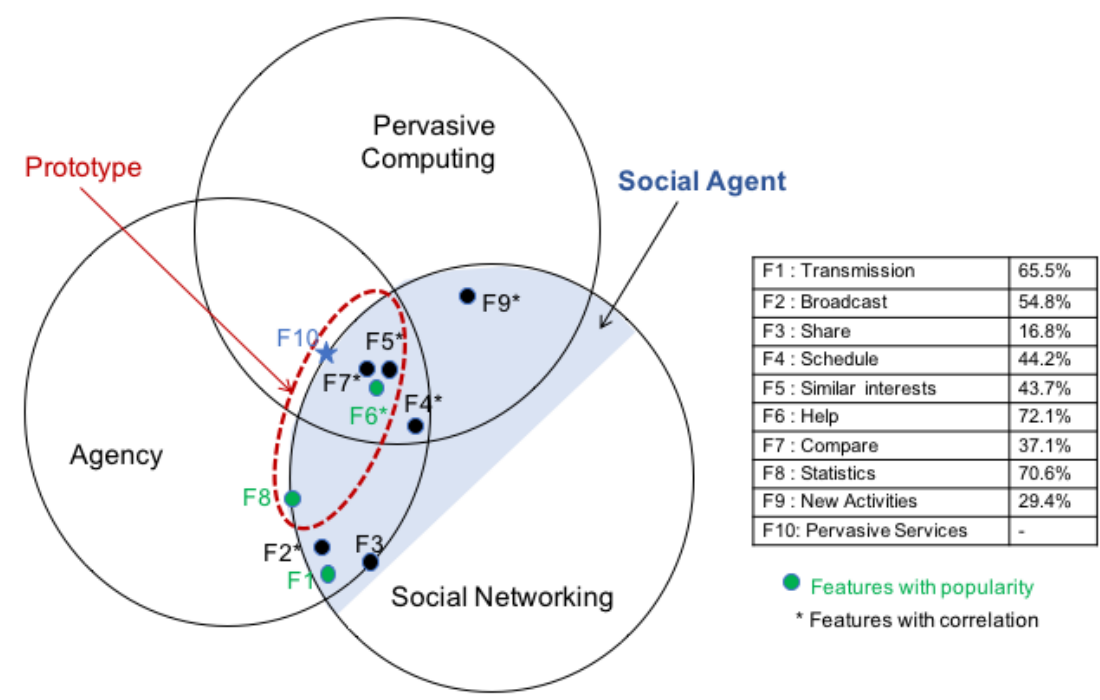

Figure 25: The domains where Social Agent is positioned, and relative evaluation results

\subsection{Added Value of Social Agent}

There is a core decision made when defining the concept of a Social Agent: digital traces defined as human activities should be the type of messages that people should exchange in order to connect ubiquitously, while a message should be meaningful and useful for them to engage in a social interaction. In other words, the question is why users should exchange messages that describe our behaviour pervasively with each other, and not typical text or media messages, like they do on existing social networking platforms. The advantages of such a decision are summarised in, but not limited to, the following list:

- The nature of pervasiveness: By sharing digital traces modelled and enhanced under the form of human activities, social agents ask for less user-input to establish pervasive social connections, meaningful for users in proximity.

- The importance of content: By reusing existing content from other platforms and domains, in the form of a meta-platform, a network of social agents may offer content to users to exchange with new social connections in proximity; users may not need motivation to create new content to be able to start using the platform.

- $\quad$ The strong semantics: By applying the anthropological notions of internalization and externalization, activity hierarchy, and tool mediation as described in Activity Theory, a properly trained system may extract useful outcomes, engage other services or enrich content with more semantics. Such platforms may be not only interoperable, but also rich in information and context.

- More sustainable business models: If not regulated or offered as infrastructure, a Social Agent may be offered together with hardware devices - which are needed to track human activities through sensors- as enhanced 
offerings, making social networking independent of advertisement-based business models that show privacy implications.

- The network effect of other devices and services ecosystem: By allowing existing or other applications to exchange interoperable activity messages, social agents may enable additional pervasive services with personalised solutions, and increase the network effect [66] of the technology. For example, based on user's current or upcoming activities, shopping coupons or recommendations may be enabled through iBeacon and digital content may adapt to media consumers' context.

- The strategic positioning: By the time current work is concluded, there is no mainstream platform that connects people in physical space over their existing activity data and preferences.

\subsection{Contribution of Our Work in Research}

Our work used some of the best practices in the research areas of Pervasive Computing, Social Networking and Social Agency, combined with the solid Activity Theory, to make Pervasive Social Networking independent of case-specific applications, and give practical results and reusable methodologies to the community. The Social Agent, as presented in section 3 and evaluated in Section 4, addressed all three research questions imposed initially. As seen in Table 10, (a) the modelling of SocAgOn developed to integrate digital traces of users with social media content enables the exchange of meaningful (i.e. for humans) pervasive social messages and unlocks further processing capabilities under Activity Theory. Then, (b) the integration of social spaces into the design of pervasive social services makes privacy a core element of Pervasive Social Networking, easier to be modelled by Social Agents and be understood by users. Finally, (c) the modified version of the TAM3 framework evaluated the high-level concept of the Social Agent under the category of Behavioural Intention to Use, separately from case-specific features grouped under the Actual Usage, in order to make questions clear for to interviewees for a concept that is not easily comprehensive (i.e. pervasiveness is by definition invisible to the user).

Table 10: Contribution of work in research questions

\begin{tabular}{|l|l|}
\hline Research Questions & Contribution \\
\hline $\begin{array}{l}\text { RQ1: Modelling digital } \\
\text { traces in pervasive social } \\
\text { networking }\end{array}$ & $\begin{array}{l}\text { Activity Theory was used to model the area of pervasive social networking and combine digital } \\
\text { traces from different resources. The generated messages may be used either as content or } \\
\text { statistical information when exchanged with other agents. }\end{array}$ \\
\hline $\begin{array}{l}\text { RQ2: Privacy in social } \\
\text { agency }\end{array}$ & $\begin{array}{l}\text { Privacy settings were perceived as a core feature of the design. By integrating user spaces into } \\
\text { the design of the social agent, agency in social networking take decisions that make users feel } \\
\text { more comfortable with. }\end{array}$ \\
\hline $\begin{array}{l}\text { RQ3: Evaluating pervasive } \\
\text { social networking }\end{array}$ & $\begin{array}{l}\text { Implemented a modified TAM model, specialised in pervasive social networking, to separate } \\
\text { evaluation of high-level concepts for pervasive social networking (i.e. expressing intention to } \\
\text { use) from specific social features (i.e. expressing actual usage). }\end{array}$ \\
\hline
\end{tabular}

In order to compare different solutions in the area of Pervasive Social Networking, a set of criteria has been generated, based on the research questions and the analysis that took place in section 2 :

- Data standardisation: Whether the suggested solution has modelled data and the way they are stored and exchanged.

- Privacy-aware social features: Whether the solution changes behaviour during social networking, based on user's privacy settings.

- Pervasive features: Whether the solution changes behaviour during social networking, based on user's context.

- User-centric design: Whether the solution is operational if a user wants to control completely the installation and manage her data.

- $\quad$ Meta-platform capabilities: Whether there are capabilities to connect with third party solutions, in order to increase quantity and quality of content in the platform.

Based on those criteria, relative work in Pervasive Social Networking is analysed and compared with the introduced concept of the Social Agent, as seen in Table 11. In particular:

- Diaspora [8] is a decentralised solution where the user is in the centre. That level of decentralisation is based on a closed-modelled platform which is free and open to install in different servers, with interfaces towards selected social media standards; however, no standardisation on the way different data resources are integrated is provided, just the server-to-server API used to push messages and control users' privacy. No special features based on users' context are provided. 
- $\quad$ Social Textiles [34] (2015) demonstrated an application for close distance, pervasive social networking, with the usage of new, smart textiles. The solution offered a dictionary of topics for users' communication, but no standardisation on integrating data was implemented, neither interfaces with external platforms. The user fully controls the social exchange, as no central platform is needed to complete an interaction. The application changes behaviour based on users' physical proximity (i.e. context), but no specific features are enabled based on user's privacy settings.

- Middle-ware, pervasive platform [50] (2007) combined physical location with social location in social networks based on users' activities, to provide a middleware for third party applications with contextual capabilities. The integration with existing social media platforms required full access on all users' network, thus no independent solutions are provided. Additionally, there is an assumption that users want to socialise in physical space only with people that are in social proximity. Users cannot control an installation, even if a decentralised architecture has been implemented, mainly for managing networking resources of the platform more effectively.

- Smart eye [75] suggested a generic architecture with components to integrate data from different domains and resources, and model pervasive computing. However, the social dimension is considered only a part of the broader architecture, and not the driving force to build pervasive social applications and create new social connections, while there is no synthesis of those different identified aspects under a common social or anthropological theory. The prototype showed no integration with other social media platforms, did not offer any privacy perspectives or any private installation, but focused mainly on users' interaction with pervasive features.

- Solid [49] (2016) is based on W3C standards and wants to put users in control of their data, while allowing other applications to build over a common pull of data. Pervasiveness is not supported natively by Solid, as each functionality is expected to be developed by special-case applications.

- $\quad$ Facebook offers lately a set of pervasive features, and allows integration with other application through its API. Lately, the OpenGraph (Site 24) standard tries to standardise the way social objects are described across the Web.

On a business level, exchanging standardised Pervasive Social Networking messages based on Activity Theory, under the proper social functionalities and rules defined by a Social Agent, may be an important step towards standardisation of modern social networking (see standardisation in Figure 26). New types of social networking applications may emerge, decentralised and rich in contextual information that existing decentralised social networks miss. By design, a Social Agent is built on a symbiotic to existing platforms way to enable users to reuse their personal data and social connections from different platforms, while it requires less time, attention and input from its owner. Additionally, Pervasive Social Networking is expected to move user's interest towards hardware solutions, where Social Agents will store personal data, and new, privacy-respectful business models may emerge, viable without ad-based revenues.

On a research level, the integration of different schemas and ontologies under Activity Theory allows reusability of knowledge from different domains and resources (i.e. devices and services), to track users' activities in the Social Agent under an enhanced modelling. The research community may reuse work done with Social Agents and experiment in different levels of the value chain for Pervasive Social Networking. Additionally, by layering social interactions based on physical proximity, the community may experiment on different features with strong pervasive and privacy capabilities. In parallel, it is very important to support serious coaching and suppressive characteristics offered by a Social Agent. Last but not least, the proposed evaluation methodology may allow researchers to evaluate different pervasive features and correlate user acceptance with the general concept of the Social Agent.

Table 11: Correlating intention to use, with proposed interactions

\begin{tabular}{|l|l|l|l|l|l|l|l|}
\hline Related Criterion & $\begin{array}{l}\text { Social } \\
\text { Agent }\end{array}$ & $\begin{array}{l}\text { Diaspora } \\
{[8]}\end{array}$ & $\begin{array}{l}\text { Social } \\
\text { Textiles } \\
{[34]}\end{array}$ & $\begin{array}{l}\text { Middle- } \\
\text { ware, } \\
\text { pervasive } \\
\text { platform [50] }\end{array}$ & $\begin{array}{l}\text { Smart } \\
\text { eye } \\
{[75]}\end{array}$ & $\begin{array}{l}\text { Solid } \\
{[49]}\end{array}$ & Facebook \\
\hline Data standardisation & Yes & No & No & No & Yes & Yes & Yes \\
\hline Privacy-aware social features & Yes & Yes & No & No & No & No & No \\
\hline Pervasive features & Yes & No & Yes & Yes & Yes & No & Yes \\
\hline User-centric design & Yes & Yes & Yes & No & No & Yes & No \\
\hline Meta-platform capabilities & Yes & Yes & No & Yes & No & Yes & Yes \\
\hline
\end{tabular}

At the end, a Social Agent does not require for social networking users to move their data away from centralised solutions (e.g. Facebook) to new, decentralised repositories, as it may be deeply integrated with existing social networking platforms. However, a broadly adopted design of a Social Agent may make the power of their digital traces and data clear to the users, and it may empower them to claim full control of their data; users may ask authorities to support their claim to download their data, be able to remove them from centralised platforms and make them 
unavailable for advertising purposes, when users do not want to receive such services. Practically speaking, a standardised modelling for combining and exchanging social networking data with contextual ones is not available at the moment; by delivering a unified, semantically-rich envelope for social messages, based on human activities, our work has contributed towards the standardisation of the Social Web. On top of that, independent platforms and applications may build an ecosystem of decentralised social networking solutions, and may force social media platforms to make available fully functional APIs that replicate their services to furtherly contribute on an open Social Web.

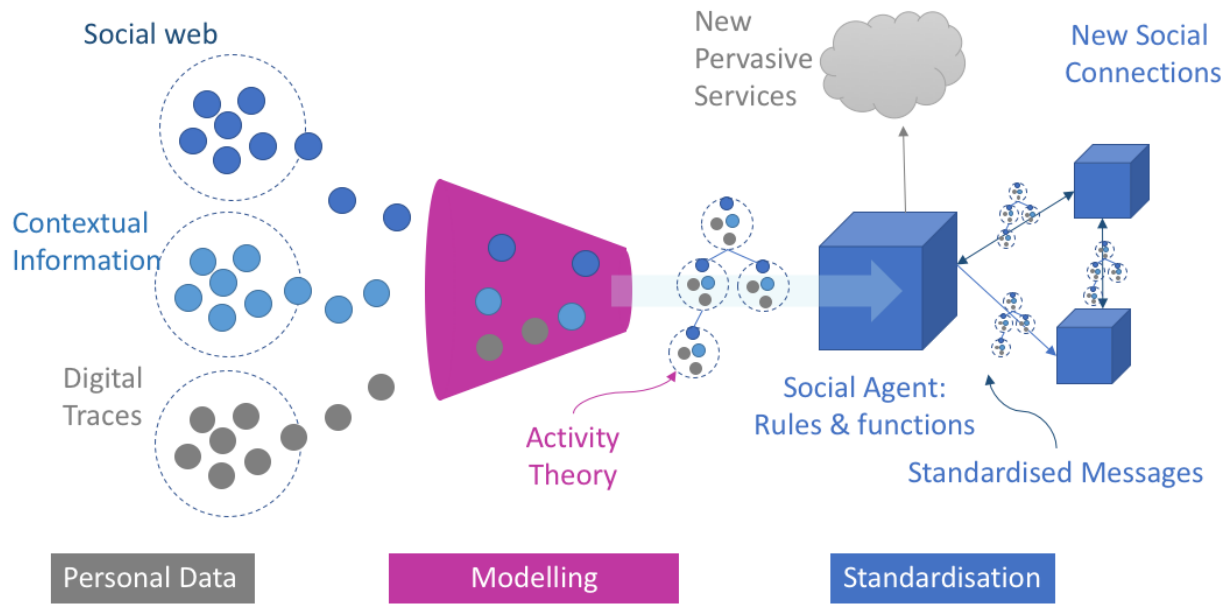

Figure 26: Modelling the area of pervasive social networking, and standardising social interactions

\section{Conclusions and Next Steps}

The presented Social Agent is a new form of digital social networking, which collaborates with existing social media platforms in the form of a meta-platform, it shows strong pervasive characteristics and a user-centric design of social features. Privacy is integrated in the core design of our approach, by exploiting the personal space of the user as context to filter interactions, while modelling for storing data and exchanging messages under the behavioural theory of Activity Theory supports extensibility and interoperability of a Social Agent, in a network of other Social Agents and an ecosystem of pervasive services in proximity.

Overall, the presented work extended the notion of digital social networking towards new dimensions, based on existing and well-established theories, and identified social trends and upcoming technologies to create the foundations of a pervasive, open and interoperable social networking ecosystem. Current work is a step forward towards the standardisation of new social applications in the form of pervasive social agents, capable of establishing close-distance communication as a new form of social networking; different social networking architectures and standards in data modelling may come together to realise the vision for an interoperable Social Web. Moreover, existing economic activities may use the aggregative knowledge of such social platforms in order to deliver better services with enhanced offerings. Thus, we consider that our work is an important contribution towards establishing the future of pervasive social networking.

In the near future, state-of-the-art technologies may enable the concept of a dedicated hardware solution for a social agent; personal agents (e.g. Amazon Echo, Google Home etc.) together with smartphones, smart watches, Wi-Fi Direct, Deep Learning and NFC may support technically the vessel where a Social Agent may be incarnated. Towards the required standardisation, more intuitive human-computer interactions may be tested, and the improved Social Agent may be connected with the middleware platforms developed already to test privacy and data analysis algorithms.

\section{Acknowledgments}

We would like to gratefully acknowledge the reviewers for the detailed and constructive feedback we received during the evaluation process, helping us improve the quality of our paper.

\section{Websites List}

Site 1: Why some social network services work and others don't - Or: the case for object-centered sociality. Jyri Engeström. April 13th, 2005 http://www.zengestrom.com/blog/2005/04/why-some-social-network-services-work-and-others-dont-or-the-case-forobject-centered-sociality.html 
Site 2: Hicon. The social Bandle.

http:/www.hiconbangle.com/

Site 3: Amico Bracelets.

http://www.amicobracelets.com/

Site 4: Personal Social Agent. www.personalsocialagent.org

Site 5: Socialwg. W3C Social Working Group.

http://www.w3.org/Social/WG

Site 6: ActivityStreams

http://activitystrea.ms/

Site 7: Schema.org.

http://schema.org/

Site 8: Solid.

https://solid.mit.edu/

Site 9: PRISM surveillance program

https://en.wikipedia.org/wiki/PRISM (surveillance program)

Site 10: UMBEL: Upper Mapping and Binding Exchange Layer

http://www.umbel.org/

Site 11: Diaspora

https://www.joindiaspora.com/

Site 12: Open Source Social Network

https://www.opensource-socialnetwork.org/

Site 13: Social Agent Ontology (SocAgOn)

http://personalsocialagent.org/about/ontology/index.html

Site 14: vCard Specifications

https://tools.ietf.org/html/rfc6350

Site 15: FoaF Specifications

http://xmlns.com/foaf/spec/

Site 16: Extensible Resource Descriptor (XRD)

http://docs.oasis-open.org/xri/xrd/v1.0/xrd-1.0.html

Site 17: W3C Social Activity

https://www.w3.org/Social/

Site 18: SIOC project

http://sioc-project.org/

Site 19: Microformats

http://microformats.org/

Site 20: Dublin Core

http://dublincore.org/

Site 21: Suggested Upper Merged Ontology (SUMO)

http://www.adampease.org/OP/

Site 22: WordNet

http://wordnet.princeton.edu/

Site 23: DBPedia

http://wiki.dbpedia.org/

Site 24: OpenGraph

http://ogp.me/

Iosif Alvertis

Evmorfia Biliri

Fenareti Lampathaki

Dimitris Askounis 
Site 25: W3C Delivery Context Ontology https://www.w3.org/TR/dcontology/

Site 26: Anonymised English version of the questionnaire https://goo.gl/forms/wio8sQhD4411jawB3

Site 27: Accenture Screenager Interactive Report, 2016

https://www.accenture.com/us-en/screenager

\section{References}

[1] M. B. Almeida and R. R. Barbosa, Ontologies in knowledge management support: A case study, Journal of the American Society for Information Science and Technology, vol. 60, no. 10, pp. 2032-2047, 2009.

[2] I. Alvertis, M. Petychakis and D. Askounis, A community-based, graph API framework to integrate and orchestrate cloud-based services, in Proceedings , 2014 IEEE/ACS 11th International Conference on Computer Systems and Applications (AICCSA), Doha, Qatar, 2014, pp. 485-492.

[3] V. Arnaboldi, M. Conti and F. Delmastro, CAMEO: A novel context-aware middleware for opportunistic mobile social networks, Pervasive and Mobile Computing, vol. 11, pp. 148-167, 2014.

[4] L. Atzori, A. lera and G. Morabito, SloT: Giving a social structure to the internet of things', IEEE Communications Letters, vol. 15, no. 11, pp. 1193-1195, 2011.

[5] L. Atzori, A. lera, G. Morabito, and M. Nitti, The social internet of things (SloT) - When social networks meet the internet of things: Concept, architecture and network characterization, Computers Networks, vol. 56, no. 16, pp. 3594-3608, 2012.

[6] E. Baumer and P. Adams, Limiting, leaving, and (re) lapsing: an exploration of Facebook non-use practices and experiences, in Proceedings of the SIGCHI Conference on Human Factors in Computing Systems Chi 2013, Paris, France, 2013, pp. 3257-3266.

[7] T. Berners-Lee, Long live the web : A call for continued open standards and neutrality, Scientific American, vol. 22, pp. 1-5, 2010.

[8] A. Bielenberg, L. Helm, A. Gentilucci, D. Stefanescu, and H. Zhang, The growth of Diaspora - A decentralized online social network in the wild, in Proceedings Computer Communications Workshops (INFOCOM WKSHPS), 2012 IEEE Conference on Computer Communications, Orlando, Florida, 2012, pp. 13-18.

[9] D. Boyd and K. Crawford, Critical questions for big data, information, Communication and Society, vol. 15, no. 5, pp. 662-679, 2012.

[10] D. M. Boyd and N. B. Ellison, Social network sites: Definition, history, and scholarship, Journal of ComputerMediated Communication, vol. 13, no. 1, pp. 210-230, 2007

[11] S. Buchegger, D. Schiöberg, L.-H. Vu, and A. Datta, PeerSoN: P2P social networking - early experiences and insights, in Proceedings of the Second ACM EuroSys Workshop on Social Network Systems - SNS '09, Nuremberg, Germany, 2009, pp. 46-52.

[12] K. Carley and A. Newell, The nature of the social agent, Journal of the Mathematical Sociology, vol. 19, no. 4, pp. 221-262, 1994.

[13] C. Castelfranchi, Modeling social action for Al agents, IJCAI International Joint Conferences on Artificial Intelligence, vol. 2, no. c, pp. 1567-1576, 1997.

[14] C. Castelfranchi, Modeling social actions for Al agents, Artificial Intelligence, vol. 103, no. January 1997, pp. 157$182,1998$.

[15] G. Cecere, F. Le Guel and N. Soulié, Perceived Internet privacy concerns on social networks in Europe, Technological Forecasting and Social Change, vol. 96, pp. 277-287, 2015.

[16] C. C. Chang, S. W. Hung, M. J. Cheng, and C. Y. Wu, Exploring the intention to continue using social networking sites: The case of Facebook, Technological Forecasting and Social Change, vol. 95, pp. 48-56, 2015.

[17] H. Chen, T. Finin and A. Joshi, The SOUPA Ontology for Pervasive Computing, Ontologies for Agents: Theory and Experiences, pp. 233-258, 2005.

[18] H. Chen, T. Finin, A. Joshi, L. Kagal, F. Perich, and D. Chakraborty, Meet the Semantic Web in Smart Spaces, IEEE Internet Computing, vol. 8, no. 6, pp. 69-79, 2004.

[19] F. T. Commission, Data brokers: A call for transparency and accountability, in Data Brokers and the Need for Transparency and Accountability, 2014, pp. 1-101.

[20] G. Conole, R. Galley and J. Culver, Frameworks for understanding the nature of interactions, networking, and community in a social networking site for academic practice, The International Review of Research in Open and Distributed Learning, Special Issue - Connectivism: Design and Delivery of Social Networked Learning, vol. 12, no. 3, pp. 119-138, 2011

[21] L. A. Cutillo, R. Molva and T. Strufe, Privacy preserving social networking through decentralization, in Proceedings WONS 2009 - 6th International Conference on Wireless On-Demand Network Systems and Services, Snowbird, Utah, 2009, pp. 145-152.

[22] S. Döweling, B. Schmidt and A. Göb, A model for the design of interactive systems based on activity theory, CSCW '12 in Proceedings of the ACM 2012 conference on Computer Supported Cooperative Work, Seattle, WA, USA, 2012, p. 539.

[23] S. Y. Esayas, A walk in to the cloud and cloudy it remains: The challenges and prospects of processing and transferring personal data, Computer Law and Security Review, vol. 28, no. 6, pp. 662-678, 2012. 
[24] T. Fong, I. Nourbakhsh and K. Dautenhahn, A survey of socially interactive robots, Robotics and Autonomous Systems, vol. 42, no. 3-4, pp. 143-166, 2003

[25] K. Gunasekera, S. Krishnaswamy, S. W. Loke, and A. Zaslavsky, Adaptation support for agent based pervasive systems, mobile and ubiquitous systems: Computing, networking, and services. MobiQuitous 2010. Lecture notes of the institute for computer sciences, Social Informatics and Telecommunications Engineering, vol. 73 LNICST, pp. 90-101, 2012.

[26] B. Guo, D. Zhang, Z. Wang, Z. Yu, and X. Zhou, Opportunistic loT: Exploring the harmonious interaction between human and the internet of things, Journal of Network and Computer Applications, vol. 36, no. 6, pp. 1531-1539, 2013.

[27] D. Helbing and S. Balietti, How to do agent-based simulations in the future: From modeling social mechanisms to emergent phenomena and interactive systems design, Santa Fe Institute, Hyde Park Road, Santa Fe, USA, Working Paper no. 11-06-024, 2011.

[28] Hellenic Statistical Authority, Survey on Information and Communication Technologies by Households and Individuals 2016 (in Greek).

[29] R. Hervás, A context model based on ontological languages : A proposal for information visualization, computer, Journal of Universal Computer Science, vol. 16, no. 12, pp. 1539-1555, 2010.

[30] P. Hu, Q. Fan and W. C. Lau. (2018, March) SNSAPI : A cross-platform middleware for rapid deployment of decentralized social networks. Cornell University Library. [Online]. Available: https://arxiv.org/abs/1403.4482

[31] L. Humphreys and R. Wilken, Social media, small businesses, and the control of information, Information, Communication \& Society, vol. 18, no. 3, pp. 295-309, 2015.

[32] R. Irfan, G. Bickler, S. U. Khan, J. Kolodziej, H. Li, D. Chen, L. Wang, K. Hayat, S. A. Madani, B. Nazir, I. A. Khan, and A. Ranjan, Survey on social networking services, IET Networks, vol. 2, no. 4, pp. 224-234, Dec. 2013.

[33] M. Kaenampornpan and E. O'Neill, Modelling context: an activity theory approach, Ambient Intelligence, pp. 367374,2004

[34] V. Kan, K. Fujii, J. Amores, C. L. Z. Jin, P. Maes, and H. Ishii, Social textiles: Social affordances and icebreaking interactions through wearable social messaging, in Proceedgins 9th International Conference on Tangible, Embedded, and Embodied Interaction, TEI 2015, Standford, CA, USA, 2015, pp. 619-624.

[35] D. Kang, B. Song, B. Yoon, Y. Lee, and Y. Park, Diffusion pattern analysis for social networking sites using smallworld network multiple influence model, Technological Forecasting and Social Change, vol. 95, pp. 73-86, 2015.

[36] V. Kaptelinin and B. A. Nardi, Activity theory: Basic concepts and applications, in Proceedgins International Conference on Human-Computer Interaction, EWHCl 1995: Human-Computer Interaction, Moscow, Russia, 1995, pp. 189-201.

[37] S. Karanasios, D. Thakker, L. Lau, D. Allen, V. Dimitrova, and A. Norman, Making sense of digital traces: An activity theory driven ontological approach, Journal of the Association for Information Science and Technology (JASIST), vol. 64, no. 12, pp. 2452-2467, 2013.

[38] Klaus B. Baerentsen and J. Trettvik, An activity theory approach to affordance, NordiCHI '02 in Proceedings of the Second Nordic Conference on Human-Computer Interaction, Aarhus, Denmark, 2002, pp.51-60.

[39] A. Kleinsmith, P. De Silva, N. Bianchi-Berthouze, L. Ardissono, P. Brna, and A. Mitrovic. (2016, February) Gumo the general User model ontology, user model. Springer Link. [Online]. Available: https://link.springer.com/chapter/ $10.1007 / 1152788658$

[40] R. Klemke, Context framework - an open approach to enhance organisational memory systems with context modelling techniques, PAKM 2000, in Proceedings of the Third International Conference on Practical Aspects of Knowledge Management, Basel, Switzerland, 2000, pp. 12-14

[41] G. Klyne, F. Reynolds, C. Woodrow, H. Ohto, J. Hjelm, M. H. Butler, and L. Tran. (2004, February) Composite capability/preference profiles (CC/PP): Structure and vocabularies 1.0, W3C recommendation. W3C. [Online]. Available: https://www.w3.org/TR/CCPP-struct-vocab/

[42] E. A. Kosmatos, N. D. Tselikas and A. C. Boucouvalas, Integrating RFIDs and smart objects into a unified internet of things architecture, Advances in Internet of Things, vol.1, pp. 5-12, 2011.

[43] N. Kourtellis, J. Finnis, P. Anderson, J. Blackburn, C. Borcea, and A. Lamnitchi, Prometheus: User-controlled P2P social data management for socially-aware applications, in Proceedings of the ACM/IFIP/USENIX 11th International Conference on Middleware, Bangalore, India, 2010, pp. 212-231.

[44] D. Kulkarni and A. Tripathi, A framework for programming robust context-aware applications, IEEE Transactions on Software Engineering, vol. 36, no. 2, 2010.

[45] J.-B. Labrune and W. Mackay, Telebeads: social network mnemonics for teenagers, IDC '06 in Proceedings of the 2006 Conference on Interaction Design and Children, Tampere, Finland, 2006, pp. 57-64

[46] P. Legris, J. Ingham and P. Collerette, Why do people use information technology? A critical review of the technology acceptance model, Information \& Management, vol. 40, no. 3, pp. 191-204, 2003.

[47] I. Leite, C. Martinho and A. Paiva, Social robots for long-term interaction: A survey, International Journal of Social Robotics, vol. 5, no. 2, pp. 291-308, 2013

[48] G. Liang and J. Cao, Social context-aware middleware: A survey, Pervasive Mobile Computing, vol. 17, no. PB, pp. 207-219, 2015.

[49] G. Lintern, An affordance-based perspective on human-machine interface design, Ecological Psychology, vol. 12 , no. 1 , pp. $65-69,2000$

[50] C. Lorenzo-Romero, M. del C. Alarcón-del-Amo and E. Constantinides, Determinants of use of social media tools in retailing sector, Journal of theoretical and applied electronic commerce research, vol. 9, no. 1, pp. 44-55, 2014. 
[51] E. Mansour, A. V. Sambra, S. Hawke, M. Zereba, S. Capadisli, A. Ghanem, A. Aboulnaga, and T. Berners-Lee, A demonstration of the solid platform for social web applications, in Proceedings on 25th International Conference of Companion World Wide Web, Montreal, Canada, 2016, pp. 223-226.

[52] S. Q. Mian, J. Riekki and H. Oinas-kukkonen, Social sensoreWeb: Towards a conceptual framework, in Proceedings Conference on E-Business, E-Services and E-Society, I3E 2016: Social Media: The Good, the Bad, and the Ugly, Swansea, United Kingdom, 2016, pp. 479-492.

[53] S. Ben Mokhtar, L. McNamara and L. Capra, A middleware service for pervasive social networking, in Proceedings M-PAC '09 of the International Workshop on Middleware for Pervasive Mobile and Embedded Computing, Urbana Champaign, Illinois 2009, pp. 1-6.

[54] M. Parameswaran and A. Whinston, Social computing: An overview, Communications of the Association for Information Systems, vol. 19, pp. 762-780, 2007.

[55] C. Parent and S. Spaccapietra, An overview of modularity, in lecture notes in computer science, Lecture Notes in Artificial Intelligence and Lecture Notes in Bioinformatics, vol. 5445, pp. 5-23, 2009.

[56] N. Peim, Activity theory and ontology, Journal of Educational Review, vol. 61, no. 2, pp. 167-180, May 2009.

[57] K. Popovic and Z. Hocenski, Cloud computing security issues and challenges, MIPRO, in Proceedings The 33rd International Convention, Opatija, Croatia, 2010, pp. 344-349.

[58] M. E. Porter and V. E. Millar. (1985, August) How information gives you competitive advantage. HBR Webinar. [Online]. Available: https://hbr.org/1985/07/how-information-gives-you-competitive-advantage

[59] M. Poveda-Villalón, M. C. Suárez-Figueroa, R. García-Castro, and A. Gómez-Pérez, A context ontology for mobile environments, in Proceedings of Workshop on Context, Information and Ontologies - CIAO 2010 Colocated with EKAW 2010, Lisbon Portugal, 2010, pp. 1-15.

[60] S. Preibusch, Privacy behaviors after snowden, Communications of the ACM, vol. 58, no. 5, pp. 48-55, 2015.

[61] D. Preuveneers, J. Van Den Bergh, D. Wagelaar, A. Georges, P. Rigole, T. Clerckx, Y. Berbers, K. Coninx, V. Jonckers, and K. De Bosschere, Towards an extensible context ontology for ambient intelligence, Ambient Intelligence, vol. 3295, pp. 148-159, 2004.

[62] D. Riboni and C. Bettini, COSAR: Hybrid reasoning for context-aware activity recognition, Personal and Ubiquitous Computing, vol. 15, no. 3, pp. 271-289, Aug. 2011

[63] D. Riboni and C. Bettini, OWL 2 modeling and reasoning with complex human activities, Pervasive Mobile Computing, vol. 7, no. 3, pp. 379-395, Jun. 2011.

[64] G. Riva, Ambient intelligence in health care, CyberPsychology \& Behavior, vol. 6, no. 3, pp. 295-300, 2003.

[65] N. D. Rodríguez, M. P. Cuéllar, J. Lilius, and M. D. Calvo-Flores, A survey on ontologies for human behavior recognition, ACM Computing Survey, vol. 46, no. 4, pp. 1-33, 2014.

[66] M. Roubroeks, J. Ham and C. Midden, When artificial social agents try to persuade people: The role of social agency on the occurrence of psychological reactance, International Journal of Social Robotics, vol. 3, no. 2, pp. 155-165, 2011

[67] D. Schuster, A. Rosi, M. Mamei, T. Springer, M. Endler, and F. Zambonelli, Pervasive social context: Taxonomy and survey, Journal ACM Transactions on Intelligent Systems and Technology (TIST), vol. 4, no. 3, p. 46, 2013.

[68] G. Siemens, Connectivism: A learning theory for the digital age, International Journal of Instructional Technology and Distance Learning (ITDL), vol. 1, pp. 1-8, 2014.

[69] P. Smaldino, C. Pickett, J. Sherman, and J. Schank, An agent-based model of social identity dynamics, Journal of Artificial Societies and Social Simulation, vol. 15, no. 4. p. 7, 2012.

[70] J. Stan, E. Egyed-Zsigmond, A. Joly, and P. Maret, A user profile ontology for situation-aware social networking, in Proceedings 3rd Workshop on Artificial Intelligence Techniques for Ambient Intelligence (AITAmI2008), Patras, Greece, 2008, pp. 51-55

[71] F. Topcu. (2011) Context modeling and reasoning techniques. SNET Seminars. [Online]. Available: https://www. snet.tu-berlin.de/fileadmin/fg220/courses/SS11/snet-project/context-modeling-and-reasoning topcu.pdf

[72] C. E. Tucker, Social networks, personalized advertising and privacy controls, Journal of Marketing Research, vol. LI, no. October, pp. 546-562, 2014

[73] C. J. Tully, Information, human activity and the nature of relevant yheories, The Computer Journal, vol. 28, no. 3, pp. 206-210, 1985.

[74] V. Venkatesh and $\mathrm{H}$. Bala, Technology acceptance model 3 and a research agenda on interventions, Decision Sciences, vol. 39, no. 2, pp. 273-315, 2008.

[75] World Economic Forum. (2011, January) Personal data: The emergence of a new asset class. Weforum. [Online]. Available: http://www3.weforum.org/docs/WEF ITTC PersonalDataNewAsset Report 2011.pdf

[76] C. Xu, S. Ryan, V. Prybutok and C. Wen, It is not for fun: An examination of social network site usage, Information Management, vol. 49, no. 5, pp. 210-217, 2012.

[77] S. S. Yau and J. Liu, Hierarchical situation modeling and reasoning for pervasive computing, in Proceedings the Fourth Software Technologies for Future Embedded and Ubiquitous Systems, 2006 and the 2006 Second International Workshop on Collaborative Computing, Integration, and Assurance. SEUS 2006/WCCIA, Gyeongju, South Korea, 2006, pp. 5-10.

[78] J. Zhou, J. Sun, K. Athukorala, D. Wijekoon, and M. Ylianttila, Pervasive social computing: Augmenting five facets of human intelligence, in Proceedings Ubiquitous Intelligence \& Computing and 7th International Conference on Autonomic \& Trusted Computing (UIC/ATC), Xian, Shaanxi, China, 2010, pp. 153-166.

[79] World Economic Forum. (206, January) White paper digital transformation of industries: In collaboration with accenture media industry. World Economic Forum. [Online]. Available: http://reports.weforum.org/digitaltransformation/wp-content/blogs.dir/94/mp/files/pages/files/digital-enterprise-narrative-final-january-2016.pdf 